\title{
FROM TRIANGULATED CATEGORIES TO CLUSTER ALGEBRAS
}

\author{
PHILIPPE CALDERO AND BERNHARD KELLER
}

\begin{abstract}
The cluster category is a triangulated category introduced for its combinatorial similarities with cluster algebras. We prove that a cluster algebra $\mathcal{A}$ of finite type can be realized as a Hall algebra, called exceptional Hall algebra, of the cluster category. This realization provides a natural basis for $\mathcal{A}$. We prove new results and formulate conjectures on 'good basis' properties, positivity, denominator theorems and toric degenerations.
\end{abstract}

\section{INTRODUCTION}

Cluster algebras were introduced by S. Fomin and A. Zelevinsky [12. They are subrings of the field $\mathbb{Q}\left(u_{1}, \ldots, u_{m}\right)$ of rational fractions in $m$ indeterminates, and defined via a set of generators constructed recursively. These generators are called cluster variables and are grouped into subsets of fixed finite cardinality called clusters. The recursion process begins with a pair $(\mathbf{x}, B)$, called a seed, where $\mathbf{x}$ is an initial cluster and $B$ is a rectangular matrix with integer coefficients.

The first aim of the theory was to provide an algebraic framework for the study of total positivity and of Lusztig/Kashiwara's canonical bases of quantum groups. The first result is the Laurent phenomenon which asserts that the cluster variables, and thus the cluster algebra they generate, are contained in the Laurent polynomial ring $\mathbb{Z}\left[u_{1}^{ \pm 1}, \ldots, u_{m}^{ \pm 1}\right]$.

Since its foundation, the theory of cluster algebras has witnessed intense activity, both, in its own foundations and in its connections with other research areas. One important aim has been to prove, as in [1, 29], ... that many algebras encountered in the theory of reductive Lie groups have (at least conjecturally) a structure of cluster algebra with an explicit seed. On the other hand, a number of recent articles have been devoted to establishing links with subjects beyond Lie theory. These links mainly rely on the combinatorics by which cluster variables are grouped into clusters. Among the subjects concerned we find Poisson geometry [17, where clusters are interpreted in terms of integrable systems, Teichmüller theory [13, where clusters are viewed as systems of local coordinates, and tilting theory [6] [4], where clusters are interpreted as sets of indecomposable factors of a tilting module.

A cluster algebra is said to be of finite type if the number of cluster variables is finite. In [14], S. Fomin and A. Zelevinsky classify the cluster algebras of finite type in terms of Dynkin diagrams. The cluster variables are then in bijection with the almost positive roots of the corresponding root system, i.e. the roots which are positive or opposite to simple roots. Note that this classification is analogous to P. Gabriel's classification of representation-finite quivers; it is also analogous to the classification of finite dimensional semisimple Lie algebras in the theory of Kac-Moody algebras.

In finite type, the combinatorics of the clusters are governed by generalized associahedra. The purpose of the decorated categories of [23 and, later, of the cluster categories of [6] 4] 9] is to offer a better understanding of these combinatorics.

Let $Q$ be a quiver whose underlying graph is a simply laced Dynkin diagram and let mod $k Q$ be the category of finite-dimensional representations of $Q$ over a field $k$. The cluster

Date: version du 09/06/2005. 
category $\mathcal{C}$ is the orbit category of the bounded derived category $\mathcal{D}^{b}(\bmod k Q)$ under the action of a canonical automorphism. Thus, it only depends on the underlying Dynkin diagram and not on the orientation of the arrows in the quiver $Q$. With hindsight, the canonical automorphism is chosen so as to extend the bijection between indecomposable $k Q$-modules and positive roots to a bijection between indecomposable objects of $\mathcal{C}$ and almost positive roots and, hence, cluster variables. By the results of [6], this bijection also induces a bijection between clusters and 'tilting objects' of $\mathcal{C}$. These 'coincidences' lead us to the following

Question 1. Can we realize a cluster algebra of finite type as a 'Hall algebra' of the corresponding category $\mathcal{C}$ ?

A first result in this direction is the cluster variable formula of 8 . This formula gives an explicit expression for the cluster variable associated with a positive root $\alpha$ corresponding to an indecomposable module $M_{\alpha}$ : The exponents of the Laurent monomials of the cluster variable $X_{\alpha}$ are provided by the homological form on $\bmod k Q$ and the coefficients are Euler characteristics of Grassmannians of submodules of $M_{\alpha}$. In the present article, we use this formula to provide a more complete answer to question 1 and to obtain structural results on the cluster algebra, some of which constitute positive answers to conjectures by Fomin and Zelevinsky. We first describe our structural results:

* Canonical basis. We obtain a $\mathbb{Z}$-basis of the cluster algebra labelled by the set of so-called exceptional objects of the category $\mathcal{C}$. The results below point to an analogy of this basis with Lusztig/Kashiwara's dual canonical bases of quantum groups.

* Positivity conjecture. We prove that the Laurent expansion of a cluster variable has positive coefficients, when the seed is associated to a quiver orientation.

* Good basis property and Toric degenerations. We prove that these bases are compatible with 'good filtrations' of the cluster algebra. This provides toric degenerations of the spectrum of cluster algebras of finite type, in the spirit of [7].

* Denominator conjecture. The formula enables us to prove the following: the denominator of the cluster variable associated to the positive root $\alpha$ in its irreducible fraction into polynomials in the $u_{i}$ 's is $\prod_{i} u_{i}^{n_{i}}$ where $\alpha=\sum_{i} n_{i} \alpha_{i}$ is the decomposition of $\alpha$ in the basis of simple roots.

Now, the main result of this article is the 'cluster multiplication theorem', Theorem 2. This result yields a more complete answer to Question 1. It provides a 'Hall algebra type' multiplication formula for the cluster algebra. The main part of the paper is devoted to the proof of this formula.

Recall that by a result of [20], the category $\mathcal{C}$ is triangulated. The cluster multiplication formula expresses the product of two cluster variables associated with objects $L$ and $N$ of the cluster category in terms of Euler characteristics of varieties of triangles with end terms $L$ and $N$. Repeated use of the formula leads to an expression of the structure constants of the cluster algebra in the basis provided by the exceptional objects in terms of Euler characteristics of varieties defined from the triangles of the category $\mathcal{C}$. Thus, the cluster algebra becomes isomorphic to what we call the 'exceptional Hall algebra' of $\mathcal{C}$.

This theorem can be compared with Peng and Xiao's theorem [24, which realizes KacMoody Lie algebras as Hall algebras of a triangulated quotient of the derived category of a hereditary category. But a closer look reveals some differences. Indeed, we use the quotient of the set of triangles $W_{N, M}^{Y}$ of the form

$$
M \rightarrow Y \rightarrow N \rightarrow M[1]
$$

by the automorphism group Aut $Y$ of the object $Y$, while Peng and Xiao use the quotient $W_{N, M}^{Y} / \operatorname{Aut}(M) \times \operatorname{Aut}(N)$. Hence, our approach is more a 'dual Hall algebra' approach as 
in Green's quantum group realization [18. Another difference is that the associativity of the multiplication is not proved a priori but results from the isomorphism with the cluster algebra.

The paper is organized as follows: Generalities and auxiliary results on triangulated categories and, in particular, on the cluster category are given in section 2 and in the appendix, where we prove the constructibility of the sets $W_{N, M}^{Y} / \operatorname{Aut}(Y)$ described above.

Section 3 deals with the cluster multiplication formula. We first reduce the proof to the case where the objects involved are indecomposable. Then the indecomposable case is solved. Here, the homology functor from $\mathcal{C}$ to the hereditary category of quiver representations plays an essential rôle. It allows us to bypass the 'triangulated geometry' of $\mathcal{C}$, which unfortunately is even out of reach of the methods of 30] 31, because the graded morphism spaces of the category $\mathcal{C}$ are not of finite total dimension. The main ingredient of the proof is the Calabi-Yau property of the cluster category, which asserts a bifunctorial duality between $\operatorname{Ext}^{1}(M, N)$ and $\operatorname{Ext}^{1}(N, M)$ for any objects $M$ and $N$.

In section 4, we use Lusztig's positivity results for canonical bases 21] 22] to prove the positivity theorem. Then we obtain the denominator theorem.

Section 5 deals with good bases for these cluster algebras. We provide a basis indexed by exceptional objects of the category $\mathcal{C}$, i.e. objects without self-extensions. The cluster variable formula yields that this basis has a 'Groebner basis' behaviour and provides toric degenerations.

The last part is concerned with conjectures for 'non hereditary' seeds of a cluster algebra of finite type. We formulate a generalization of the cluster variable formula, and other conjectures which would follow from it, such as results on positivity, simplicial fans ... . We close the article with a positivity conjecture for the multiplication rule of the exceptional Hall algebra.

Acknowledgments. The authors thank F. Chapoton and C. Geiss for stimulating discussions. They are grateful to A. Zelevinsky for pointing out an error in an earlier version of this article.

\section{The Cluster CATEgory}

2.1. Let $\Delta$ be a simply laced Dynkin diagram and $Q$ a quiver with underlying graph $\Delta$. We denote the set of vertices of $Q$ by $Q_{0}$ and the set of arrows by $Q_{1}$. Let $k$ be a field. We denote by $k Q$ the path algebra of $Q$ and by $\bmod k Q$ the category of finitely generated right $k Q$-modules. For $i \in Q_{0}$, we denote by $P_{i}$ the associated indecomposable projective $k Q$-module and by $S_{i}$ the associated simple module. The Grothendieck group $\mathrm{G}_{0}(\bmod k Q)$ is free abelian on the classes $\left[S_{i}\right], i \in Q_{0}$, and is thus isomorphic to $\mathbb{Z}^{n}$, where $n$ is the number of vertices of $Q$. For any object $M$ in $\bmod k Q$, the dimension vector of $M$, denoted by $\underline{\operatorname{dim}}(M)$, is the class of $M$ in $\mathrm{G}_{0}(\bmod k Q)$.

Recall that the category $\bmod k Q$ is hereditary, i.e. we have $\operatorname{Ext}^{2}(M, N)=0$ for any objects $M, N$ in $\bmod k Q$. For all $M, N$ in $\bmod k Q$, we put

$$
[M, N]^{0}=\operatorname{dim} \operatorname{Hom}(M, N),[M, N]^{1}=\operatorname{dim} \operatorname{Ext}^{1}(M, N),\langle M, N\rangle=[M, N]^{0}-[M, N]^{1} .
$$

In the sequel, for any additive category $\mathcal{F}$, we denote by ind $(\mathcal{F})$ the subcategory of $\mathcal{F}$ formed by a system of representatives of the isomorphism classes of indecomposable objects in $\mathcal{F}$. We know that there exists a partial ordering $\preceq_{Q}$, also denoted by $\preceq$, on ind $(\bmod k Q)$ such that

$$
[M, N]^{0} \neq 0 \Rightarrow M \preceq N, \quad M, N \in \operatorname{ind}(\bmod k Q) .
$$

Denote by $r$ be the cardinality of ind $(\bmod k Q)$. We fix a numbering $Z_{k}, 1 \leq k \leq r$, of the objects in ind $\bmod k Q)$ which is compatible with the ordering. 
We recall from [27] 28] that there is a canonical $\mathbb{Z}$-linear category from which mod $k Q$ is obtained by base change.

2.2. Denote by $\mathcal{D}=\mathcal{D}_{Q}=\mathcal{D}^{b}(\bmod k Q)$ the bounded derived category of the category of finitely generated $k Q$-modules and by $S$ its shift functor $M \mapsto M[1]$. As shown in [19, the category $\mathcal{D}$ is a Krull-Schmidt category, and, up to canonical triangle equivalence, it only depends on the underlying graph $\Delta$ of $Q$. We identify the category $\bmod k Q$ with the full subcategory of $\mathcal{D}$ formed by the complexes whose homology is concentrated in degree 0 . We simply call 'modules' the objects in this subcategory. The indecomposable objects of $\mathcal{D}$ are the $S^{j} Z_{k}, j \in \mathbb{Z}, 1 \leq k \leq r$.

We still denote by $\underline{\operatorname{dim}}(M) \in \mathrm{G}_{0}(\mathcal{D})$ the dimension vector of an object $M$ of $\mathcal{D}$ in the Grothendieck group of $\mathcal{D}$.

Let $\tau$ be the AR-translation of $\mathcal{D}$. It is the autoequivalence of $\mathcal{D}$ characterized by the Auslander-Reiten formula:

$$
\operatorname{Ext}_{\mathcal{D}}^{1}(N, M) \simeq D \operatorname{Hom}_{\mathcal{D}}(M, \tau N),
$$

where $M, N$ are any objects in $\mathcal{D}$ and where $D$ is the functor which takes a vector space to its dual. The AR-translation $\tau$ is a triangle equivalence and therefore induces an automorphism of the Grothendieck group of $\mathcal{D}$. If we identify this group with the root lattice of the corresponding root system, the Auslander-Reiten translation corresponds to the Coxeter transformation, $c f$. [3] [15].

We now consider the orbit category $\mathcal{D} / F$, where $F$ is the autoequivalence $S \circ \tau^{-1}$. The objects of the category $\mathcal{C}=\mathcal{C}_{Q}=\mathcal{D} / F$ are the objects of $\mathcal{D}$ and the morphisms are defined by

$$
\operatorname{Hom}_{\mathcal{D} / F}(M, N)=\coprod_{i \in \mathbb{Z}} \operatorname{Hom}_{\mathcal{D}}\left(F^{i} M, N\right) .
$$

The category $\mathcal{C}_{Q}$ was defined in [6], $c f$. also [9] for the $\mathrm{A}_{n}$-case. It is the so-called cluster category. Like the derived category $\mathcal{D}$, up to canonical equivalence, the cluster category $\mathcal{C}_{Q}$ only depends on $\Delta$ and not on the orientation of the quiver $Q$.

2.3. We now review the basic properties of the category $\mathcal{C}$. The first two points of the following theorem were proved in [20] and and the last two points in [6].

Theorem 1. (i) The category $\mathcal{C}$ is triangulated and

(ii) the natural functor $\pi: \mathcal{D} \rightarrow \mathcal{C}$ is a triangle functor.

(iii) The category $\mathcal{C}$ is a Krull-Schmidt category and

(iv) we have $\operatorname{End}_{\mathcal{C}}(M)=k$ for any indecomposable object $M$ of $\mathcal{C}$.

The shift functor of the triangulated category $\mathcal{C}$ will still be denoted by $S$. Often, we will omit the functor $\pi$ from the notations. With this convention, the objects in

$$
\text { ind } \bmod k Q \cup\left\{S P_{i}, 1 \leq i \leq n\right\}
$$

form a set of representatives for the indecomposables of $\mathcal{C}$, as shown in Proposition 1.6 of [6]. By formula [2.1] we have, for all objects $M, N$ of $\mathcal{D}$, that

$$
\operatorname{Ext}_{\mathcal{D}}^{1}(M, N) \simeq D \operatorname{Hom}_{\mathcal{D}}(N, \tau M) \simeq D \operatorname{Hom}_{\mathcal{D}}\left(\tau^{-1} N, M\right) \simeq D \operatorname{Ext}^{1}(F N, M) .
$$

Hence, the category $\mathcal{C}$ is Calabi-Yau of CY-dimension 1 , which means that the functor Ext ${ }^{1}$ is symmetric in the following sense:

$$
\operatorname{Ext}_{\mathcal{C}}^{1}(M, N) \simeq D \operatorname{Ext}_{\mathcal{C}}^{1}(N, M) .
$$

In other words, there is an (almost) canonical non degenerate bifunctorial pairing

$$
\phi: \operatorname{Ext}_{\mathcal{C}}^{1}(M, N) \times \operatorname{Ext}_{\mathcal{C}}^{1}(N, M) \rightarrow k .
$$


2.4. In this section, we study the analogy between the (triangulated) category $\mathcal{C}$ and the (abelian) category $\bmod k Q$. We will see that it can be useful to view $\mathcal{C}$ as glued together from copies of mod $k Q^{\prime}$, where $Q^{\prime}$ runs through the set of orientations of $\Delta$.

By the previous section, each object $M$ of $\mathcal{C}$ can be uniquely decomposed in the following way:

$$
M=M_{0} \oplus S P_{M},
$$

where $M_{0}$ is the image under $\pi$ of a 'module' in $\mathcal{D}$, and where $S P_{M}$ is the image of the shift of a projective module. We will say that an object $M$ of $\mathcal{C}$ is a module if $M=M_{0}$, and that $M$ is the shift of a projective module if $M=S P_{M}$.

The module $M_{0}$ can be recovered using the functor

$$
H^{0}=\operatorname{Hom}_{\mathcal{C}}\left(k Q_{k Q}, ?\right): \mathcal{C} \rightarrow \bmod k Q .
$$

Indeed, we have

$$
H^{0}(M)=H^{0}\left(M_{0}\right) \oplus H^{0}\left(S P_{M}\right)=\operatorname{Hom}_{\bmod k Q}\left(k Q_{k Q}, M_{0}\right) \oplus \operatorname{Hom}_{\mathcal{C}}\left(\oplus_{i} P_{i}, S P_{M}\right)=M_{0},
$$

as the last factor is zero. The functor $H^{0}$ is a homological functor, i.e. it maps triangles in $\mathcal{C}$ to long exact sequences of $k Q$-modules.

We will deduce the following proposition from Proposition 1.7 of $[6]$.

Proposition 1. Let $M, N$ be indecomposable $k Q$-modules. Then

(i) $\operatorname{Ext}_{\mathcal{C}}^{1}(M, N)=\operatorname{Ext}_{k Q}^{1}(M, N) \amalg \operatorname{Ext}_{k Q}^{1}(N, M)$ and at least one of the two direct factors vanishes.

(ii) any short exact sequence of $k Q$-modules $0 \longrightarrow M \stackrel{i}{\longrightarrow} Y \stackrel{p}{\longrightarrow} N \longrightarrow 0$ provides a (unique) triangle $M \stackrel{i}{\longrightarrow} Y \stackrel{p}{\longrightarrow} N \longrightarrow S M$ in $\mathcal{C}$,

(iii) if $M \preceq N$ and if there exists a triangle $M \stackrel{i}{\longrightarrow} Y \stackrel{p}{\longrightarrow} N \longrightarrow S M$ in $\mathcal{C}$, then $Y$ is also a module and there exists a short exact sequence of $k Q$-modules $0 \longrightarrow M \stackrel{i}{\longrightarrow} Y \stackrel{p}{\longrightarrow} N \longrightarrow 0$. Moreover, if this sequence is non split, the modules $M$ and $N$ are non isomorphic and are not isomorphic to indecomposable factors of $Y$.

Proof. Point (i) is proved in proposition 1.7 of 6 . For points (ii) and (iii), we may assume that $\operatorname{Ext}_{k Q}^{1}(N, M)$ does not vanish. This implies that there is a non zero morphism from $M$ to $\tau N$ and thus we have $M \preceq N$. If $\operatorname{Ext}_{k Q}^{1}(N, M)$ was also non zero, we would also have $M \preceq N$, hence $M=N$ and $\operatorname{Ext}_{k Q}(M, N)=0$, a contradiction. Now it follows from point (i) that the canonical map

$$
\operatorname{Ext}_{k Q}^{1}(M, N) \rightarrow \operatorname{Ext}_{\mathcal{C}}^{1}(M, N)
$$

is bijective. Points (ii) and the first assertion of (iii) therefore follow from the bijection between elements of $\mathrm{Ext}^{1}$ and classes of short exact sequences in mod $k Q$ respectively triangles in $\mathcal{C}$. For the last assertion of (iii), we first note that $M$ is not isomorphic to $N$ because no indecomposable module has selfextensions. Now since the sequence is non split, the map $Y \rightarrow N$ factors through the middle term of the AR-sequence ending in $N$. Therefore each indecomposable factor of $Y$ strictly precedes $N$. Dually, $M$ must precede each indecomposable factor of $Y$.

The following lemma is useful to understand extensions between indecomposable objects of $\mathcal{C}$ when the situation does not fit into the framework of the proposition above. 
Lemma 1. Let $M \rightarrow Y \rightarrow N \rightarrow S M$ be a non split triangle, where $M, N$ are indecomposable objects of $\mathcal{C}$. Suppose that there exists no orientation $Q^{\prime}$ of $\Delta$ such that $M, N$ are simultaneously $k Q^{\prime}$-modules with $M \preceq Q^{\prime} N$ via the embedding $\bmod k Q^{\prime} \rightarrow \mathcal{D} \rightarrow \mathcal{C}$. Then, $N=S M$ and $Y=0$.

Proof. Using the AR-translation, it is always possible to choose an embedding of $\bmod k Q$ in $\mathcal{D}$ such that $M$ is a projective module. By changing orientations, we can suppose that $M$ is simple projective, say $P_{i}$. By the hypothesis of the lemma, $N$ is not a module, and, as $N$ is indecomposable, $N$ is the shift of a projective $S P_{j}$. As the triangle above is non split, the morphism $\varepsilon: N \rightarrow S M$ is non zero. So there exists a non zero morphism from $P_{j}$ to $P_{i}$. The assumption on $P_{i}$ implies $j=i$. Hence, $N=S M$ and the morphism $\varepsilon$ is an isomorphism. This forces $Y$ to be zero.

2.5. In this section, we give properties of group actions on triangles in $\mathcal{C}$. Actually, most of them are general facts valid in Krull-Schmidt triangulated categories.

Let $Y$ be an object of $\mathcal{C}$ and $Y=\coprod_{j} Y_{j}$ be a decomposition into isotypical components. Suppose that, for each $j$, the object $Y_{j}$ is the sum of $n_{j}$ copies of an indecomposable. By Theorem 1(iv), the endomorphism algebra of each $Y_{j}$ is isomorphic to a matrix algebra over $k$. Therefore, the radical $R$ of $\operatorname{End}_{\mathcal{C}}(Y)$ is formed by the endomorphisms $f$ all of whose components $f_{j j}: Y_{j} \rightarrow Y_{j}$ vanish. We obtain the decomposition

$$
\operatorname{End}_{\mathcal{C}}(Y)=\prod_{j} M_{n_{j}}(k) \oplus R
$$

Therefore, the group $\operatorname{Aut}(Y)$ of invertible elements of $\operatorname{End}_{\mathcal{C}}(Y)$ is isomorphic to $L(Y) \ltimes U$, where $L(Y)=\prod_{j} \mathrm{GL}_{n_{j}}(k)$ and where $U$ is the unipotent group $1+R$. We denote by $W_{N, M}^{Y}$ the set of triples $(i, p, \eta)$ of morphisms such that $M \stackrel{i}{\longrightarrow} Y \stackrel{p}{\longrightarrow} N \stackrel{\eta}{\longrightarrow} S M$ is a triangle. The group Aut $(Y)$ acts on $W_{N, M}^{Y}$ by $g \cdot(i, p, \eta)=\left(g i, p g^{-1}, \eta\right)$. There also exists an action of $\operatorname{Aut}(M) \times \operatorname{Aut}(N)$ on $W_{N, M}^{Y}$ given by $(g, h) \cdot(i, p, \eta)=\left(i g^{-1}, h p, S g \eta h^{-1}\right)$. In particular, we can define an action of the group $k^{*}$ on $W_{N, M}^{Y}$ given by $\lambda .(i, p, \eta)=$ $\left(\lambda^{-1} i, p, \lambda \eta\right)$.

Lemma 2. For any objects $M, Y, N$ of $\mathcal{C}$, the group $\operatorname{Aut}(Y)$ acts on $W_{N, M}^{Y}$ with unipotent stabilizers.

Proof. Fix a triple $(i, p, \eta)$ in $W_{N, M}^{Y}$ and let $g$ in the stabilizer of $(i, p, \eta)$ for the action of Aut $(Y)$. We can draw the following commuting diagram

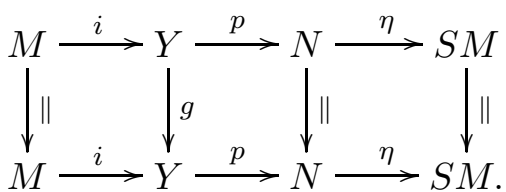

As the identity $I$ lies in the stabilizer, we get the solid part of the commutative diagram

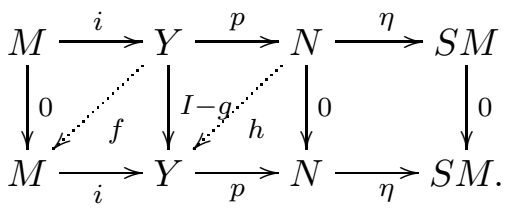

The long exact sequence obtained by applying $\operatorname{Hom}_{\mathcal{C}}(Y$, ?) to the bottom triangle shows that there is a morphism $f$ with if $=I-g$. Similarly, there is morphism $h$ with $h p=I-g$. Therefore, we have $(I-g)^{2}=(h p)(i f)=0$. This proves our assertion. 
Consider the third projection $p_{3}: W_{N, M}^{Y} \rightarrow \operatorname{Ext}^{1}(N, M)$. Define $\operatorname{Ext}^{1}(N, M)_{Y}$ to be the image of $W_{N, M}^{Y}$ under $p_{3}$. For any $\eta$ in $\operatorname{Ext}^{1}(M, N)_{Y}$, the group Aut $(Y)$ clearly acts on $p_{3}^{-1}(\eta)$.

Lemma 3. For any $\eta$ in $\operatorname{Ext}^{1}(N, M)_{Y}$, the action of Aut $(Y)$ on $p_{3}^{-1}(\eta)$ is transitive and has unipotent stabilizers.

Proof. Let $(i, p, \eta)$ and $\left(i^{\prime}, p^{\prime}, \eta\right)$ be in $p_{3}^{-1}(\eta)$. Then, by [32, TR3], there exists a morphism $f$ which makes the following diagram commutative.

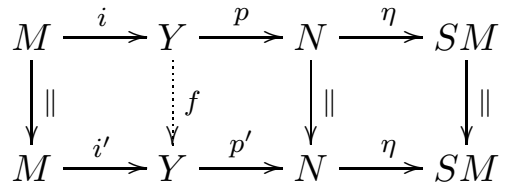

By the 5-Lemma, $f$ is invertible. The last assertion is Lemma 2.

2.6. Let $K_{0}^{\text {split }}(\mathcal{C})$ be the Grothendieck group of the underlying additive category of $\mathcal{C}$, i.e. the free abelian group generated by the isomorphism classes of indecomposable objects of $\mathcal{C}$. For each object $Y$ of $\mathcal{C}$, we still denote by $Y$ the corresponding element of $K_{0}^{\text {split }}(\mathcal{C})$.

For a variety $X$, we define $\chi_{c}(X)$ to be the Euler-Poincaré characteristic of the étale cohomology with proper support of $X$, i.e. we have

$$
\chi_{c}(X)=\sum_{i=0}^{\infty}(-1)^{i} \operatorname{dim} H_{c}^{i}\left(X, \overline{\mathbb{Q}}_{l}\right) .
$$

Let $M, N, Y$ be objects of $\mathcal{C}$. By section 17 the subset $\left.\operatorname{Ext}^{1}(M, N)_{Y}\right)$ of the vector space $\operatorname{Ext}^{1}(M, N)$ is constructible. Clearly it is conic. Thus its projectivization $\left.\mathbb{P} \operatorname{Ext}^{1}(M, N)_{Y}\right)$ is a variety and has a well-defined Euler characteristic $\chi_{c}\left(\mathbb{P} \operatorname{Ext}^{1}(M, N)_{Y}\right)$.

For any pair $\left(Z_{j}, Z_{i}\right)$ of indecomposable object of $\mathcal{C}$, we call elementary vector associated to this pair any element $Z_{i}+Z_{j}-Y_{i j}$ in $K_{0}^{s p l i t}(\mathcal{C})$ such that $Y_{i j}$ is the middle term of a non split triangle $Z_{i} \longrightarrow Y_{i j} \longrightarrow Z_{j} \longrightarrow S Z_{i}$ in $\mathcal{C}$. In this case, the number $c_{i j}=\chi_{c}\left(\operatorname{Ext}^{1}\left(\mathbb{P}\left(Z_{j}, Z_{i}\right)_{Y_{i j}}\right)\right.$ is called the multiplicity number of the elementary vector. The following proposition is an analogue of Theorem 3.3 in [1].

Proposition 2. Let $M, N, Y$ be any objects of $\mathcal{C}$ such that $Y \neq M \oplus N$.

(i) If $M+N-Y$ is an elementary vector associated to the pair $\left(Z_{j}, Z_{i}\right)$, then we have

$$
\chi_{c}\left(\mathbb{P} \operatorname{Ext}^{1}(N, M)_{Y}\right)=z_{i} z_{j} c_{i j}
$$

where $z_{j}$, resp. $z_{i}$, is the multiplicity of the indecomposable component $Z_{j}$, resp. $Z_{i}$, in $N$, resp. $M$, and where $c_{i j}$ is multiplicity number associated to the elementary vector.

(ii) If $M+N-Y$ is not an elementary vector, then

$$
\chi_{c}\left(\mathbb{P} \operatorname{Ext}^{1}(N, M)_{Y}\right)=0 .
$$

Proof. Let $A, B$ be two objects of $\mathcal{C}$. Since $\mathcal{C}$ is triangulated, for each morphism $i$ from $A$ to $B$, there is a triangle $A \stackrel{i}{\longrightarrow} B \longrightarrow C \longrightarrow S A$ and the object $C$ is unique up to (non unique) isomorphism. Hence, we have a partition

$$
\operatorname{Hom}_{\mathcal{C}}(A, B)=\coprod_{C} \operatorname{Hom}_{\mathcal{C}}(A, B)_{C},
$$


where $C$ runs through the isomorphism classes of $\mathcal{C}$ and

$\operatorname{Hom}_{\mathcal{C}}(A, B)_{C}=\left\{i \in \operatorname{Hom}_{\mathcal{C}}(A, B) \mid\right.$ There is a triangle $\left.A \stackrel{i}{\longrightarrow} B \longrightarrow C \longrightarrow S A\right\}$.

Now, by [32, TR2] and Lemma 1.3 of [19], we have $\operatorname{Ext}^{1}(N, M)_{Y}=\operatorname{Hom}(N, S M)_{S Y}$. This implies that

$$
\operatorname{Ext}^{1}(A, B)=\coprod_{C} \operatorname{Ext}^{1}(A, B)_{C}
$$

Now we prove (i) and (ii). Denote by $M=\oplus_{i} m_{i} Z_{i}$ and $N=\oplus_{j} n_{j} Z_{j}$ the decompositions of $M$ and $N$ into indecomposable objects. Consider the action of $G:=\operatorname{Aut}(M) \times \operatorname{Aut}(N)$ on $\mathbb{P} \operatorname{Ext}^{1}(N, M)$. The group $G$ contains as a subgroup

$$
H=\prod_{i} \operatorname{Aut}\left(m_{i} Z_{i}\right) \times \prod_{j} \operatorname{Aut}\left(n_{j} Z_{j}\right)=\prod_{i} \mathrm{GL}_{m_{i}} \times \prod_{j} \mathrm{GL}_{n_{j}} .
$$

Under the isomorphism

$$
\operatorname{Ext}^{1}(N, M)=\oplus_{(i, j)} \operatorname{Ext}^{1}\left(Z_{j}, Z_{i}\right) \otimes M_{n_{j} \times m_{j}},
$$

the action of $H$ on $\operatorname{Ext}^{1}(N, M)$ corresponds to the product of the canonical actions of the $\mathrm{GL}_{m_{i}} \times \mathrm{GL}_{n_{j}}$ on matrices. Let $T$ be the torus of $G$ corresponding to the diagonal matrices. We have $T \subset H \subset G$ and $T$ acts on $\mathbb{P} \operatorname{Ext}^{1}(N, M)$ with set of invariants

$$
\mathbb{P} \operatorname{Ext}^{1}(N, M)^{T}=\coprod_{(i, j)} \coprod_{r, s} \mathbb{P} \operatorname{Ext}^{1}\left(Z_{j}, Z_{i}\right) \otimes k E_{r s},
$$

where, for fixed $(i, j)$, the $E_{r s}, 1 \leq r \leq n_{j}, 1 \leq s \leq m_{i}$, are the elementary matrices in $M_{n_{j} \times m_{i}}$.

Set $\mathcal{X}:=\mathbb{P} \operatorname{Ext}^{1}(N, M)_{Y}$. As $G$ acts on $\mathcal{X}$, we have an action of the torus $T$ and

$$
\mathcal{X}^{T}=\mathbb{P} \operatorname{Ext}^{1}(N, M)^{T} \cap \mathcal{X} .
$$

It follows from Białynicki-Birula's results [2] that we have $\chi_{c}(\mathcal{X})=\chi_{c}\left(\mathcal{X}^{T}\right)$. We will compute $\chi_{c}\left(\mathcal{X}^{T}\right)$. If $\mathcal{X}^{T}$ is empty, we have $\chi_{c}(\mathcal{X})=\chi_{c}\left(\mathcal{X}^{T}\right)=0$. Let us suppose from now on that $\mathcal{X}^{T}$ is non empty. Let the element $[\eta] \otimes E_{r s}$ be in $\mathcal{X}^{T}$ with $[\eta] \in \mathbb{P} \operatorname{Ext}^{1}\left(Z_{j}, Z_{i}\right)$. As $G$, hence $H$, acts on $\mathcal{X}$, the element $[\eta] \otimes E_{r^{\prime}} s^{\prime}$ is also in $\mathcal{X}^{T}$ for any $r^{\prime}, s^{\prime}, 1 \leq r^{\prime} \leq n_{j}$, $1 \leq s^{\prime} \leq m_{i}$. Now, by [2.2, there exists a unique object $Y_{i j}$ such that $[\eta]$ belongs to $\mathbb{P} \operatorname{Ext}^{1}\left(Z_{j}, Z_{i}\right)_{Y_{i j}}$. And so, in $K_{0}^{\text {split }}(\mathcal{C})$, we have

$$
Y=M+N-Z_{i}-Z_{j}+Y_{i j}
$$

This implies that $M+N-Y$ is an elementary vector. Moreover, for any element $\left[\eta^{\prime}\right]$ of $\mathbb{P} \operatorname{Ext}^{1}\left(Z_{j}, Z_{i}\right)_{Y_{i j}}$, the element $\left[\eta^{\prime}\right] \otimes E_{k l}$ belongs to $\mathbb{P} \operatorname{Ext}^{1}(N, M)_{Y^{\prime}}$, where

$$
Y^{\prime}=M+N-Z_{i}-Z_{j}+Y_{i j}=Y \text {. }
$$

Thus, $Y^{\prime}$ is isomorphic to $Y$. So we have proved the inclusion

$$
\coprod_{k, l} \mathbb{P} \operatorname{Ext}^{1}\left(Z_{j}, Z_{i}\right)_{Y_{i j}} \otimes E_{k l} \subset \mathcal{X}^{T} .
$$

The proposition will follow if we prove the reverse inclusion. Suppose that $\left[\eta^{\prime}\right]$ belongs to $\mathbb{P} \operatorname{Ext}^{1}\left(Z_{j}, Z_{i}\right)_{Y_{i j}^{\prime}}$ with $\left[\eta^{\prime}\right] \otimes E_{k l}$ belonging to $\mathcal{X}^{T}$. Then, we obtain $Y=M+N-Z_{i}-Z_{j}+Y_{i j}^{\prime}$, which implies $Y_{i j}^{\prime}=Y_{i j}$. Now, in order to finish the proof of the reverse inclusion, it is sufficient to prove that if we have two triangles

$$
M \longrightarrow Y \longrightarrow N \stackrel{\eta}{\longrightarrow} S M, M \longrightarrow Y \longrightarrow N \stackrel{\eta^{\prime}}{\longrightarrow} S M
$$


with $\eta \in \operatorname{Ext}^{1}\left(Z_{j}, Z_{i}\right)$ and $\eta^{\prime} \in \operatorname{Ext}^{1}\left(Z_{j^{\prime}}, Z_{i^{\prime}}\right)$, then $(i, j)$ equals $\left(i^{\prime}, j^{\prime}\right)$. Let us prove this fact. The first triangle implies that $M+N-Y=Z_{j}+Z_{i}-Y_{i j}$ for some object $Y_{i j}$. The second one implies that $M+N-Y=Z_{j^{\prime}}+Z_{i^{\prime}}-Y_{i^{\prime} j^{\prime}}$ for some object $Y_{i^{\prime} j^{\prime}}$. Comparing both equalities in $K_{0}^{\text {split }}(\mathcal{C})$ we get $Z_{j}+Z_{i}-Y_{i j}=Z_{j^{\prime}}+Z_{i^{\prime}}-Y_{i^{\prime} j^{\prime}}$. It follows from Lemma 1 and part (iii) of Proposition 1 that the modules $Z_{j}$ and $Z_{i}$ are non isomorphic and are not isomorphic to indecomposable factors of $Y_{i j}$, and similarly for $Z_{i^{\prime}}, Z_{j^{\prime}}$ and $Y_{i^{\prime} j^{\prime}}$. Therefore, the signs in the equality imply that $(i, j)=\left(i^{\prime}, j^{\prime}\right)$ or $(i, j)=\left(j^{\prime}, i^{\prime}\right)$. In the second case, we have $Y_{i j}=Y_{i^{\prime} j^{\prime}}$, but it follows from Lemma 1 and part (iii) of Proposition 1 that we cannot simultaneously have two triangles

$$
Z_{i} \longrightarrow Y_{i j} \longrightarrow Z_{j} \stackrel{\eta}{\longrightarrow} S Z_{i} \text {, and } Z_{j} \longrightarrow Y_{i j} \longrightarrow Z_{i} \stackrel{\eta^{\prime}}{\longrightarrow} S Z_{j} \text {. }
$$

Hence, we must have $(i, j)=\left(i^{\prime}, j^{\prime}\right)$ and we are done.

\section{The Cluster MULtipliCATION THEOREM}

3.1. We now present the main theorem. The subsections 3.2, 3.3, 3.4 are devoted to the proof of the theorem.

For any $k Q$-module $M$, let $\operatorname{Gr}(M)$ be the Grassmannian of $k Q$-submodules of $M$, and let $\operatorname{Gr}_{e}(M)$ be the $e$-Grassmannian of $M$, i.e. the variety of submodules of $M$ with dimension vector $e$.

Following [8] we define

$$
X_{?}: \operatorname{obj}\left(\mathcal{C}_{Q}\right) \rightarrow \mathbb{Q}\left(x_{1}, \ldots, x_{n}\right), M \mapsto X_{M}
$$

to be the unique map with the following properties:

(i) $X_{M}$ only depends on the isomorphism class of $M$,

(ii) we have

$$
X_{M \oplus N}=X_{M} X_{N}
$$

for all $M, N$ of $\mathcal{C}_{Q}$,

(iii) if $M$ is isomorphic to $S P_{i}$ for the $i t h$ indecomposable projective $P_{i}$, we have

$$
X_{M}=x_{i},
$$

(iv) if $M$ is the image in $\mathcal{C}_{Q}$ of an indecomposable $k Q$-module, we have

$$
X_{M}=\sum_{e} \chi_{c}\left(\mathrm{Gr}_{e}(M)\right) x^{\tau(e)-\underline{\operatorname{dim}} M+e},
$$

where $\tau$ is the Auslander-Reiten translation on the Grothendieck group of $\mathcal{D}_{Q}$ and, for $v \in \mathbb{Z}^{n}$, we put

$$
x^{v}=\prod_{i=1}^{n} x_{i}^{\left\langle\underline{\operatorname{dim}} S_{i}, v\right\rangle} .
$$

From now on, we write $\operatorname{Ext}^{1}(N, M)$ for $\operatorname{Ext}_{\mathcal{C}}^{1}(N, M)$ for any objects $N, M$ of $\mathcal{C}$.

Theorem 2. For any objects $M, N$ of $\mathcal{C}$, we have

(i) If $\operatorname{Ext}^{1}(N, M)=0$, then $X_{N} X_{M}=X_{N \oplus M}$,

(ii) If $\operatorname{Ext}^{1}(N, M) \neq 0$, then

$$
\chi_{c}\left(\mathbb{P} \operatorname{Ext}^{1}(N, M)\right) X_{N} X_{M}=\sum_{Y}\left(\chi_{c}\left(\mathbb{P} \operatorname{Ext}^{1}(N, M)_{Y}\right)+\chi_{c}\left(\mathbb{P} \operatorname{Ext}^{1}(M, N)_{Y}\right)\right) X_{Y},
$$

where $Y$ runs through the isoclasses of $\mathcal{C}$.

Note that the first part is true by the definition of the map $M \mapsto X_{M}$. 
3.2. We prove here that Theorem 2 is true if it is true for indecomposable $k Q$-modules $M$, $N$, and for any orientation $Q$ of $\Delta$. So we suppose the following: For all indecomposable $k Q$-modules $Z_{i}, Z_{j}$ with $\operatorname{Ext}^{1}\left(Z_{j}, Z_{i}\right) \neq 0$, we have

$$
(*) \quad \chi_{c}\left(\mathbb{P} \operatorname{Ext}^{1}\left(Z_{j}, Z_{i}\right)\right) X_{Z_{j}} X_{Z_{i}}=\sum_{Y_{i j}}\left(\chi_{c}\left(\mathbb{P} \operatorname{Ext}^{1}\left(Z_{j}, Z_{i}\right)_{Y_{i j}}\right)+\chi_{c}\left(\mathbb{P} \operatorname{Ext}^{1}\left(Z_{i}, Z_{j}\right)_{Y_{i j}}\right)\right) X_{Y_{i j}} .
$$

By Proposition 2.6 of [ $[$ ], the formula above is also true for all indecomposable objects $Z_{i}, Z_{j}$ such that $Z_{i}=\tau Z_{j}=S Z_{j}$. Hence, by Lemma 1 our hypothesis implies that the theorem is true for all indecomposable objects $M$ and $N$ of $\mathcal{C}_{Q}$.

Suppose now that $M$ and $N$ are arbitrary objects of $\mathcal{C}$, with decompositions into indecomposables $M=\oplus m_{i} Z_{i}, N=\oplus n_{j} Z_{j}$. Then we have

$$
\chi_{c}\left(\mathbb{P}\left(\operatorname{Ext}^{1}(N, M)\right)\right)=\sum m_{i} n_{j} \chi_{c}\left(\mathbb{P} \operatorname{Ext}^{1}\left(Z_{j}, Z_{i}\right)\right) .
$$

Moreover, if an object $Y$ is such that $\chi_{c}\left(\mathbb{P} \operatorname{Ext}^{1}(N, M)_{Y}\right)$ is non zero, then, by Proposition 2. there exist unique objects $Z_{i}, Z_{j}, Y_{i j}$ such that

$$
\chi_{c}\left(\mathbb{P} \operatorname{Ext}^{1}(N, M)_{Y}\right)=m_{i} n_{j} \chi_{c}\left(\mathbb{P} \operatorname{Ext}^{1}\left(Z_{j}, Z_{i}\right)_{Y_{i j}}\right)
$$

and $Y_{i j} \oplus\left(n_{j}-1\right) Z_{j} \oplus\left(m_{i}-1\right) M_{i}=Y$. Hence, by multiplying all equalities (*) by $m_{i} n_{j} X_{\left(n_{j}-1\right) Z_{j} \oplus\left(m_{i}-1\right) M_{i}}$ and adding all these equalities, we obtain the formula of Theorem 2

3.3. From now on, in the rest of the proof of theorem 2, we can suppose that $M$ and $N$ are indecomposable $k Q$-modules.

Suppose $X_{\mathbb{F}_{q}}$ is a family of sets indexed by all finite fields $\mathbb{F}_{q}$ such that the cardinality $\left|X_{\mathbb{F}_{q}}\right|$ is a polynomial $P_{X}$ in $q$ with integer coefficients. In particular, we will consider the case where $X_{\mathbb{Z}}$ is a variety defined over $\mathbb{Z}$ and, for any field $k, X_{k}$ is the corresponding variety defined by base change. In this case, we will set $\chi_{1}(X):=P_{X}(1)$. Then it is a consequence of the Grothendieck trace formula, see [25], [8, Lemma 3.5], that $\chi_{1}(X)$ is exactly the Euler characteristic $\chi_{c}\left(X_{\mathbb{C}}\right)$ of $X_{\mathbb{C}}$. We apply this to prove the following Lemma.

Lemma 4. For any indecomposable modules $M, N$, any module $Y$, and any dimension vector $e$, we have $\chi_{c}\left(\mathbb{P} \operatorname{Ext}^{1}(M, N)_{Y}\right)=\chi_{1}\left(\mathbb{P} \operatorname{Ext}^{1}(M, N)_{Y}\right)$ and $\chi_{c}\left(\operatorname{Gr}_{e}(M)\right)=\chi_{1}\left(\operatorname{Gr}_{e}(M)\right)$.

Proof. By part (i) of Proposition [1] we have

$$
\operatorname{Ext}^{1}(M, N)_{Y}=\operatorname{Ext}_{k Q}^{1}(M, N)_{Y} \text { or } \operatorname{Ext}^{1}(M, N)_{Y}=\operatorname{Ext}_{k Q}^{1}(N, M)_{Y}
$$

Therefore, we know from [26] that this variety is obtained by base change from a variety defined over $\mathbb{Z}$ and that the cardinality of its set of $\mathbb{F}_{q}$-points is polynomial in $q$. The corresponding facts for $\operatorname{Gr}_{e}(M)$ were shown in [8].

This lemma allows us to work in the remainder of the section in the case where the base field $k$ is the finite field $\mathbb{F}_{q}$ with $q$ elements.

Let $N, M$ be indecomposable $k Q$-modules such that

$$
0 \neq \operatorname{Ext}_{k Q}^{1}(N, M)
$$

This implies that $\operatorname{Ext}_{k Q}^{1}(M, N)$ vanishes and hence that

$$
\operatorname{Ext}_{\mathcal{C}_{Q}}^{1}(N, M)=\operatorname{Ext}_{k Q}^{1}(N, M) \oplus D \operatorname{Ext}_{k Q}^{1}(M, N)=\operatorname{Ext}_{k Q}^{1}(N, M) .
$$

We can suppose that we are in this case. 
For a module $Y$, the group $\operatorname{Aut}(Y)$ acts naturally on $\operatorname{Gr}(Y)$ and this action stabilizes the subvarieties $\operatorname{Gr}(Y)$. If $Y$ is any object, we define the action of $L(Y):=\operatorname{Aut}\left(H^{0}(Y)\right) \times$ Aut $\left(S P_{Y}\right)$ on $\operatorname{Gr}\left(H^{0}(Y)\right)$ by letting the second factor act trivially.

If a group $G$ acts on the sets $X$ and $Y$, then $G$ acts on $X \times Y$ by $g \cdot(x, y)=(g \cdot x, g \cdot y)$. We define as usual the set of orbits $X \times_{G} Y$ for this action. By section 2.5. we obtain:

Lemma 5. Let $M, N, Y$ be objects of $\mathcal{C}$. Suppose that $\operatorname{Ext}^{1}(N, M)_{Y} \neq 0$. Then we have

$$
\chi_{1}\left(W_{N, M}^{Y} \times{ }_{\left(L(Y) \times k^{*}\right)} \operatorname{Gr}\left(H^{0}(Y)\right)=\chi_{1}\left(\mathbb{P} \operatorname{Ext}^{1}(N, M)_{Y} \times \operatorname{Gr}\left(H^{0}(Y)\right) .\right.\right.
$$

If $Y$ is a module, then we have

$$
\chi_{1}\left(W_{N, M}^{Y} \times{ }_{\left(\operatorname{Aut}(Y) \times k^{*}\right)} \operatorname{Gr}(Y)\right)=\chi_{1}\left(\mathbb{P} \operatorname{Ext}^{1}(N, M)_{Y} \times \operatorname{Gr}(Y)\right) .
$$

Note that we have

$$
\chi_{c}\left(\mathbb{P} \operatorname{Ext}^{1}(N, M)\right)=\operatorname{dim} \operatorname{Ext}^{1}(N, M) .
$$

so that we could 'simplify' the left hand side of the main formula in Theorem 2 Now, with the help of Lemma 5. we see that the specialization of the claimed formula at $x_{i}=1$, $1 \leq i \leq n$, is just the equality of the Euler characteristics $\chi_{1}$ of the following varieties

$$
L=\mathbb{P} \operatorname{Ext}^{1}(N, M) \times \operatorname{Gr}(N) \times \operatorname{Gr}(M)
$$

and

$$
R=\left(\coprod_{Y} W_{N, M}^{Y} \times_{\text {Aut }(Y) \times k^{*}} \operatorname{Gr}(Y)\right) \coprod\left(\coprod_{Y} W_{M, N}^{Y} \times_{L(Y) \times k^{*}} \operatorname{Gr}\left(H^{0}(Y)\right)\right) .
$$

More precisely, the left hand side of the equality claimed in the theorem is a sum of terms

$$
\chi_{c}\left(\left(\mathbb{P} \operatorname{Ext}^{1}(N, M) \times \operatorname{Gr}_{e}(N)\right) \times \operatorname{Gr}_{f}(M)\right) x^{\tau e-\underline{\operatorname{dim}} N+e+\tau f-\underline{\operatorname{dim}} M+f .} .
$$

Now the variety

$$
L(e, f)=\left(\mathbb{P} \operatorname{Ext}^{1}(N, M) \times \operatorname{Gr}_{e}(N)\right) \times \operatorname{Gr}_{f}(M)
$$

is the union of the subvariety $L_{1}(e, f)$ consisting of all those triples $\left([\varepsilon], N^{\prime}, M^{\prime}\right)$ such that there is a diagram of $k Q$-modules $\left(\right.$ since $\operatorname{Ext}_{k Q}^{1}(N, M)=\operatorname{Ext}_{\mathcal{C}_{Q}}(N, M)$ )

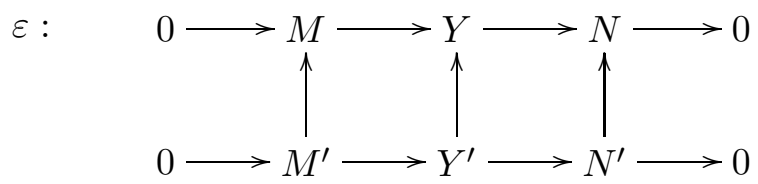

and its complement $L_{2}(e, f)$. By Proposition 3 below, we see that the cardinality of $L_{2}(e, f)$ and hence of $L_{1}(e, f)$ over $\mathbb{F}_{q}$ is polynomial in $q$, so the numbers $\chi_{1}\left(L_{i}(e, f)\right), i=1,2$ make sense.

Now, the term

$$
\left.\chi_{c}\left(\mathbb{P} \operatorname{Ext}^{1}(N, M) \times \operatorname{Gr}_{e}(N)\right) \times \operatorname{Gr}_{f}(M)\right) x^{\tau e-\underline{\operatorname{dim}} N+e+\tau f-\underline{\operatorname{dim}} M+f}
$$

is the sum of

$$
\chi_{1}\left(L_{1}(e, f)\right) x^{\tau e-\underline{\operatorname{dim}} N+e+\tau f-\underline{\operatorname{dim}} M+f}
$$

and

$$
\chi_{1}\left(L_{2}(e, f)\right) x^{\tau e-\underline{\operatorname{dim}} N+e+\tau f-\underline{\operatorname{dim}} M+f} .
$$

Now we examine the right hand side of the equality of the theorem: First note that since we have $\operatorname{Ext}_{k Q}^{1}(N, M)=\operatorname{Ext}_{\mathcal{C}_{Q}}^{1}(N, M)$, the set $\operatorname{Ext}^{1}(N, M)_{Y}$ is empty if $Y$ does not occur as the middle term of an extension

$$
0 \rightarrow M \rightarrow Y \rightarrow N \rightarrow 0
$$


in the category of modules. Therefore, we have

$$
\chi_{c}\left(\mathbb{P} \operatorname{Ext}^{1}(N, M)_{Y}\right) X_{Y}=\sum_{g} \chi_{c}\left(\mathbb{P} \operatorname{Ext}^{1}(N, M)_{Y} \times \operatorname{Gr}_{g}(Y)\right) x^{\tau g-\underline{\operatorname{dim}} Y+g} .
$$

For non empty $\operatorname{Ext}^{1}(N, M)_{Y}$, by Proposition 1, one defines a map

$$
W_{N, M}^{Y} \times \mathrm{Gr}_{g}(Y) \rightarrow \coprod_{e+f=g} L_{1}(e, f),\left(i, p, \varepsilon, Y^{\prime}\right) \mapsto\left([\varepsilon], Y^{\prime} \cap i(M), p\left(Y^{\prime}\right)\right)
$$

This map descends to a map $W_{N, M}^{Y} \times$ Aut $(Y) \times k^{*} \operatorname{Gr}_{g}(Y) \rightarrow \coprod_{e+f=g} L_{1}(e, f)$ which is surjective with fibers which are affine spaces. One obtains the equality of the Euler characteristics and therefore

$$
\chi_{c}\left(\mathbb{P} \operatorname{Ext}^{1}(N, M)_{Y}\right) X_{Y}=\sum_{e, f} \chi_{1}\left(L_{1}(e, f)\right) x^{\tau e-\underline{\operatorname{dim}} N+e+\tau f-\underline{\operatorname{dim}} M+f} .
$$

It remains to be proved that

$$
\sum_{e, f} \chi_{1}\left(L_{2}(e, f)\right) x^{\tau e-\underline{\operatorname{dim}} N+e+\tau f-\underline{\operatorname{dim}} M+f}=\sum_{Y} \chi_{c}\left(\mathbb{P} \operatorname{Ext}^{1}(M, N)_{Y}\right) X_{Y} .
$$

For this, we need a characterization of the points in $L_{2}(e, f)$. Let $\left([\varepsilon], N^{\prime}, M^{\prime}\right)$ be a point of

$$
L(e, f)=\mathbb{P} \operatorname{Ext}^{1}(N, M) \times \operatorname{Gr}_{e}(N) \times \operatorname{Gr}_{f}(M)
$$

Let

$$
\phi: \operatorname{Ext}_{\mathcal{C}_{Q}}^{1}(M, N) \times \operatorname{Ext}_{\mathcal{C}_{Q}}^{1}(N, M) \rightarrow k
$$

be the (almost) canonical duality pairing. Let

$$
\beta: \operatorname{Ext}^{1}\left(M, N^{\prime}\right) \rightarrow \operatorname{Ext}^{1}(M, N) \oplus \operatorname{Ext}^{1}\left(M^{\prime}, N^{\prime}\right)
$$

be the map whose components are induced by the inclusions $M^{\prime} \subset M$ and $N^{\prime} \subset N$.

Proposition 3. The following are equivalent

(i) $\left([\varepsilon], N^{\prime}, M^{\prime}\right)$ belongs to $L_{2}(e, f)$.

(ii) $\varepsilon$ is not orthogonal to $\operatorname{Ext}^{1}(M, N) \cap \operatorname{Im} \beta$.

(iii) There is an $\eta \in \operatorname{Ext}^{1}(M, N)$ such that $\phi(\eta \varepsilon) \neq 0$ and such that, if

$$
N \stackrel{i}{\longrightarrow} Y \stackrel{p}{\longrightarrow} M \stackrel{\eta}{\longrightarrow} S N
$$

is a triangle of $\mathcal{C}_{Q}$, then there is a diagram of $k Q$-modules

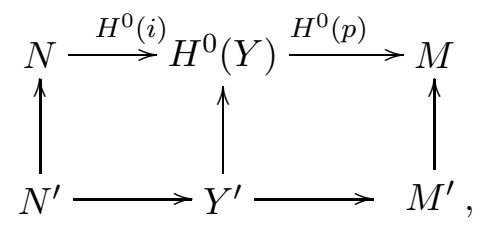

where $Y^{\prime}$ is a submodule of $H^{0}(Y), N^{\prime}$ is the preimage of $Y^{\prime}$ and $M^{\prime}$ the image of $Y^{\prime}$.

The proposition will be proved in section 3.4. We continue the proof of the theorem. Recall the equality we have to prove:

$$
\sum_{e, f} \chi_{1}\left(L_{2}(e, f)\right) x^{\tau e-\underline{\operatorname{dim}} N+e+\tau f-\underline{\operatorname{dim}} M+f}=\sum_{Y} \chi_{c}\left(\mathbb{P} \operatorname{Ext}^{1}(M, N)_{Y}\right) X_{Y} .
$$


Each object $Y$ of $\mathcal{C}_{Q}$ is isomorphic to the sum of $H^{0}(Y)$ with a module $S P_{Y}$ for some projective $P_{Y}$. With this notation, the right hand side equals

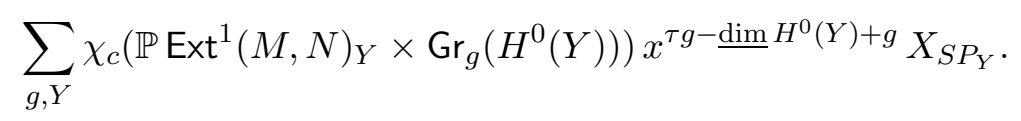

To prove the equality, we will define a correspondence $C$ between

$$
L_{2}=\coprod_{e, f} L_{2}(e, f) \subset \mathbb{P} \operatorname{Ext}^{1}(N, M) \times \operatorname{Gr}(N) \times \operatorname{Gr}(M)
$$

and

$$
R_{2}=\coprod_{Y, g} R_{2}(Y, g), \text { where } R_{2}(Y, g)=W_{M, N}^{Y} \times_{L(Y) \times k^{*}} \operatorname{Gr}_{g}\left(H^{0}(Y)\right)
$$

Namely, the correspondence $C \subset L_{2} \times R_{2}$ is formed by all pairs consisting of a point $\left([\varepsilon], M^{\prime}, N^{\prime}\right)$ of $L_{2}$ and a point $\left(i, p, \eta, Y^{\prime}\right)$ of $R_{2}$ such that $\phi(\eta \varepsilon) \neq 0, N^{\prime}=H^{0}(i)^{-1}\left(Y^{\prime}\right)$, $M^{\prime}=H^{0}(p)\left(Y^{\prime}\right)$. Note that in this situation, we have a diagram

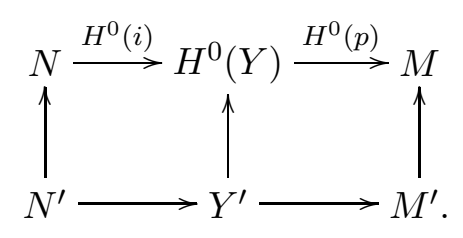

We say that a variety $X$ is an extension of affine spaces if there is a vector space $V$ and a surjective morphism $X \rightarrow V$ whose fibers are affine spaces of constant dimension.

\section{Proposition 4.}

a) The projection $p_{1}: C \rightarrow L_{2}$ is surjective and its fibers are extensions of affine spaces.

b) The projection $p_{2}: C \rightarrow R_{2}$ is surjective and its fibers are affine spaces of constant dimension.

c) If the pair formed by $\left([\varepsilon], M^{\prime}, N^{\prime}\right)$ and $\left(i, p, \eta, Y^{\prime}\right)$ belongs to $C$, then

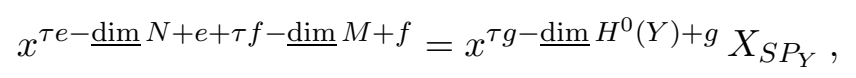

where $Y=H^{0}(Y) \oplus S P_{Y}, P_{Y}$ is projective, $e=\underline{\operatorname{dim}} M^{\prime}, f=\underline{\operatorname{dim}} N^{\prime}, g=\underline{\operatorname{dim}} Y^{\prime}$.

This proposition, which will be proved in section 3.5. allows us to conclude: Indeed, the variety $C$ is the disjoint union of the

$$
C_{e, f, Y, g}=p_{1}^{-1}\left(L_{2}(e, f)\right) \cap p_{2}^{-1}\left(R_{2}(Y, g)\right)
$$

by parts a) and b) of the proposition, for each non empty $C_{e, f, Y, g}$, we have equality of cardinalities on $\mathbb{F}_{q}$

$$
\#\left(L_{2}(e, f)\right)=\sum_{Y, g} q^{m_{e, f, Y, g}} \#\left(C_{e, f, Y, g}\right) \text { and } \sum_{e, f} q^{n_{e, f, Y, g}} \#\left(C_{e, f, Y, g}\right)=\chi_{c}\left(R_{2}(Y, g)\right)
$$


where $m_{e, f, Y, g}$ and $n_{e, f, Y, g}$ are (non positive) integers. Moreover, if $C_{e, f, Y, g}$ is non empty, then we have the equality of part c) of the proposition. Thus we have

$$
\begin{aligned}
\sum_{e, f} \#\left(L_{2}(e, f)\right) x^{\tau e-\underline{\operatorname{dim}} N+e+\tau f-\underline{\operatorname{dim}} M+f} & =\sum_{e, f, Y, g} q^{m_{e, f, Y, g}} \#\left(C_{e, f, Y, g}\right) x^{\tau e-\underline{\operatorname{dim}} N+e+\tau f-\underline{\operatorname{dim}} M+f} \\
& =\sum_{e, f, Y, g} q^{m_{e, f, Y, g}} \#\left(C_{e, f, Y, g}\right) x^{\tau g-\underline{\operatorname{dim}} H^{0}(Y)+g} X_{S P_{Y}} \\
& =\sum_{e, f, Y, g} q^{n_{e, f, Y, g}} \#\left(C_{e, f, Y, g}\right) x^{\tau g-\underline{\operatorname{dim}} H^{0}(Y)+g} X_{S P_{Y}}+Z \\
& =\sum_{Y, g} \#\left(R_{2}(Y, g)\right) x^{\tau g-\underline{\operatorname{dim}} H^{0}(Y)+g} X_{S P_{Y}}+Z,
\end{aligned}
$$

where $Z$ is a term which vanishes at $q=1$. Hence,

$$
\begin{aligned}
\sum_{e, f} \chi_{1}\left(L_{2}(e, f)\right) x^{\tau e-\underline{\operatorname{dim}} N+e+\tau f-\underline{\operatorname{dim}} M+f} & =\sum_{Y, g} \chi_{1}\left(R_{2}(Y, g)\right) x^{\tau g-\underline{\operatorname{dim}} H^{0}(Y)+g} X_{S P_{Y}} \\
& =\sum_{Y} \chi_{c}\left(\mathbb{P} \operatorname{Ext}^{1}(M, N)_{Y}\right) X_{Y} .
\end{aligned}
$$

3.4. This section is devoted to the proof of Proposition 3

Before proving the equivalence of (i) and (ii), we need some preparation: consider the diagram

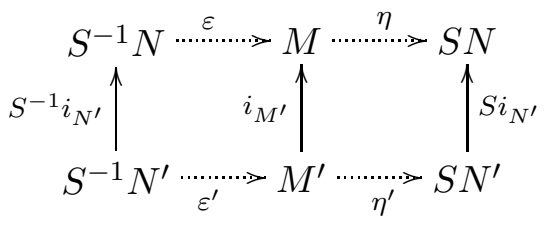

Note that (i) holds if and only if there is no $\varepsilon^{\prime}$ which makes the left hand square commutative. We formalize this as follows: The diagram yields two complexes

$$
\begin{gathered}
\left(S^{-1} N, M^{\prime}\right) \stackrel{\alpha^{\prime}}{\longrightarrow}\left(S^{-1} N, M\right) \oplus\left(S^{-1} N^{\prime}, M^{\prime}\right) \stackrel{\beta^{\prime}}{\longrightarrow}\left(S^{-1} N^{\prime}, M\right) \\
\left(M^{\prime}, S N\right) \longleftarrow \alpha \\
\longleftarrow
\end{gathered}
$$

where we write $($,$) for \operatorname{Hom}_{\mathcal{C}_{Q}}($,$) and where$

$\alpha^{\prime}=\left[\begin{array}{c}\left(i_{M^{\prime}}\right)_{*} \\ \left(S^{-1} i_{N^{\prime}}\right)^{*}\end{array}\right], \beta^{\prime}=\left[\left(S^{-1} i_{N^{\prime}}\right)^{*},-\left(i_{M}^{\prime}\right)_{*}\right], \alpha=\left[\left(i_{M^{\prime}}\right)_{*},\left(S i_{N^{\prime}}\right)^{*}\right], \beta=\left[\begin{array}{c}\left(S i_{N^{\prime}}\right)^{*} \\ -\left(i_{M^{\prime}}\right)_{*}\end{array}\right]$.

The two complexes are in duality via the pairings

$$
(\eta, \varepsilon) \mapsto \phi(\eta \circ \varepsilon) \text { and }\left(\eta^{\prime}, \varepsilon^{\prime}\right) \mapsto \phi^{\prime}\left(\eta^{\prime} \circ \varepsilon^{\prime}\right)
$$

given by the (almost) canonical forms

$$
\phi: \operatorname{Hom}_{\mathcal{C}_{Q}}\left(S^{-1} N, S N\right) \rightarrow k \text { and } \phi^{\prime}: \operatorname{Hom}_{\mathcal{C}_{Q}}\left(S^{-1} N^{\prime}, S N^{\prime}\right) \rightarrow k .
$$

Now let us prove the equivalence of (i) and (ii). Let $p$ denote the projection

$$
\left(S^{-1} N, M\right) \oplus\left(S^{-1} N^{\prime}, M^{\prime}\right) \rightarrow\left(S^{-1} N, M\right) .
$$


Then (i) says that $\varepsilon$ does not belong to $p\left(\operatorname{ker} \beta^{\prime}\right)$. This holds if and only if $\varepsilon$ is not orthogonal to the orthogonal of $p\left(\operatorname{ker} \beta^{\prime}\right)$ in $(M, S N) \oplus\left(M^{\prime}, S N^{\prime}\right)$. Now the orthogonal of the image of the map

$$
\operatorname{ker} \beta^{\prime} \longrightarrow\left(S^{-1} N, M\right) \oplus\left(S^{-1} N^{\prime}, M^{\prime}\right) \stackrel{p}{\longrightarrow}\left(S^{-1}, M\right)
$$

is the kernel of its transpose

$$
\operatorname{cok} \beta \longleftarrow(M, S N) \oplus\left(M^{\prime}, S N^{\prime}\right) \longleftarrow(M, S N)
$$

and this is precisely $(M, S N) \cap \operatorname{Im} \beta$. So (i) holds if and only if $\varepsilon$ is not orthogonal to $(M, S N) \cap \operatorname{Im} \beta$, which means that (i) and (ii) are equivalent.

Let us prove that (ii) implies (iii). We choose a morphism $f: M \rightarrow S N^{\prime}$ such that $\beta(f)$ belongs to $\operatorname{Ext}^{1}(M, N)$ and is not orthogonal to $\varepsilon$. This means that we have

$$
\left(S i_{N^{\prime}}\right) \circ f=\eta, f \circ i_{M^{\prime}}=0, \phi(\eta \varepsilon) \neq 0 .
$$

Now we form triangles on $\eta: M \rightarrow S N$ and $0: M^{\prime} \rightarrow S N^{\prime}$. Thanks to the fact that $\eta i_{M^{\prime}}=0$, we have a morphism of triangles

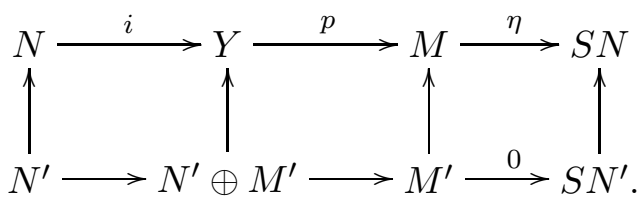

By applying $H^{0}$ we obtain a morphism of long exact sequences

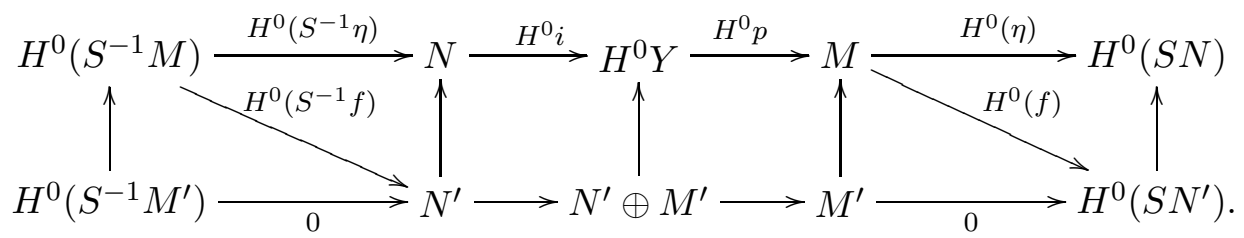

Now we let $Y^{\prime}$ be the image of $N^{\prime} \oplus M^{\prime}$ in $H^{0} Y$. An easy diagram chase then shows that $N^{\prime}$ is the preimage of $Y^{\prime}$ under $H^{0} i$ and $M^{\prime}$ is the image of $Y^{\prime}$ under $H^{0} p$.

Let us prove that (iii) implies (ii). We are given $\eta$ such that $\phi(\eta \varepsilon) \neq 0$, a triangle of $\mathcal{C}_{Q}$

$$
N \stackrel{i}{\longrightarrow} Y \stackrel{p}{\longrightarrow} M \stackrel{\eta}{\longrightarrow} S N
$$

and a diagram of $k Q$-modules

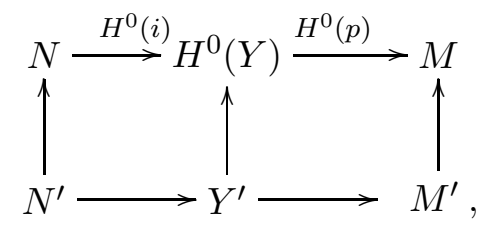

where $Y^{\prime}$ is a submodule of $H^{0}(Y), N^{\prime}$ is the preimage of $Y^{\prime}$ and $M^{\prime}$ the image of $Y^{\prime}$. We will show that $\eta$ belongs to $\operatorname{Ext}^{1}(M, N) \cap \operatorname{Im} \beta$. For this we consider the larger diagram

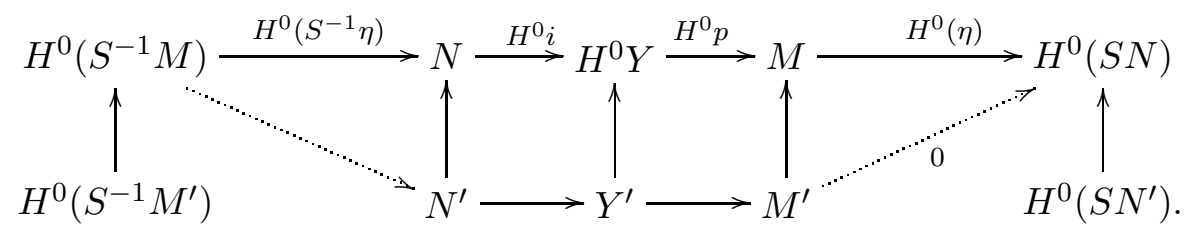

Here $H^{0}\left(S^{-1} \eta\right)$ factors through $N^{\prime}$ since its image is contained in the kernel of $H^{0} i$, which is contained in $N^{\prime}$. Moreover, $H^{0} \eta$ vanishes on $M^{\prime}$ since $M^{\prime}$ is contained in the image of 
$H^{0} p$. Now recall that $M$ and $N$ are indecomposable $k Q$-modules and $\operatorname{Ext}_{k Q}^{1}(N, M) \neq 0$. Therefore $M$ is non injective and so $S^{-1} M=\tau^{-1} M$ is still a module (and not just an object of $\mathcal{C}_{Q}$ ) and similarly, $N$ is non projective and so $S N=\tau N$ is still a module. Moreover, $M^{\prime}$ cannot have an injective direct factor (since that would also be an injective direct factor of $M$ ) and so $S^{-1} M^{\prime}=\tau^{-1} M^{\prime}$ is still a module and similarly $S N^{\prime}=\tau N^{\prime}$ is still a module.

We would like to show that $\eta \in \operatorname{Hom}_{\mathcal{C}_{Q}}(M, \tau N)$ comes from a morphism of modules. For this, recall that we have

$$
\operatorname{Hom}_{\mathcal{C}_{Q}}(U, V)=\sum_{n \in \mathbb{Z}} \operatorname{Hom}_{\mathcal{D}^{b}(k Q)}\left(F^{n} U, V\right)
$$

for arbitrary modules $U, V$, where $F=\tau^{-1} S$. Moreover, if $U$ and $V$ are indecomposable and either $U$ or $V$ does not lie on a cycle of $\mathcal{D}^{b}(k Q)$, then by part b) of proposition 1.5 of [6], there is at most one $n \in \mathbb{Z}$ such that

$$
\operatorname{Hom}_{\mathcal{D}^{b}(k Q)}\left(F^{n} U, V\right) \neq 0 .
$$

We know that indecomposable postprojective $k Q$-modules do not lie on cycles of $\mathcal{D}^{b}(k Q)$. Thus if $U$ and $V$ are indecomposable and one of them is postprojective, we have

$$
\operatorname{Hom}_{k Q}(U, V) \neq 0 \Rightarrow \operatorname{Hom}_{k Q}(U, V) \stackrel{\sim}{\rightarrow} \operatorname{Hom}_{\mathcal{C}_{Q}}(U, V) .
$$

In the case where $Q$ is a Dynkin quiver (which we assume), all modules are postprojective.

Now $M$ and $\tau N$ are indecomposable and we have

$$
\operatorname{Hom}_{k Q}(M, \tau N)=D \operatorname{Ext}_{k Q}^{1}(N, M) \neq 0
$$

and hence

$$
\operatorname{Hom}_{k Q}(M, \tau N)=\operatorname{Hom}_{\mathcal{C}_{Q}}(M, \tau N) .
$$

In particular, $\eta$ comes from a morphism of modules so that we have $\eta=H^{0} \eta$. Since $H^{0} \eta$ vanishes on $M^{\prime} \subset M$, the composition of $\eta$ with $i_{M^{\prime}}$ vanishes. It remains to be shown that $\eta$ factors through $S N^{\prime}$. Now $\tau^{-1} M$ and $N$ are also indecomposable and we have

$$
\operatorname{Hom}_{k Q}\left(\tau^{-1} M, N\right)=D \operatorname{Ext}_{k Q}^{1}(N, M) \neq 0
$$

and hence

$$
\operatorname{Hom}_{k Q}\left(\tau^{-1} M, N\right)=\operatorname{Hom}_{\mathcal{C}_{Q}}\left(\tau^{-1} M, N\right)
$$

Thus $\tau^{-1} \eta$ comes from a morphism of modules and $\tau^{-1} \eta=H^{0}\left(\tau^{-1} \eta\right)$. Since $H^{0}\left(\tau^{-1} \eta\right)$ factors as $i_{N^{\prime}} f$ for a morphism $f: \tau^{-1} M \rightarrow N^{\prime}$, we have $\tau^{-1}(\eta)=i_{N^{\prime}} f$ and therefore

$$
\eta=\tau\left(i_{N^{\prime}}\right) \tau f=\left(S i_{N^{\prime}}\right)(S f) .
$$

3.5. We now give a proof of Proposition 4

We prove part a). The projection $p_{1}: C \rightarrow L_{2}$ is surjective by the equivalence between (i) and (iii) in Proposition [3, Let $\left([\varepsilon], M^{\prime}, N^{\prime}\right)$ be in $L_{2}$ and pick an element of $p_{2}\left(p_{1}^{-1}\left([\varepsilon], M^{\prime}, N^{\prime}\right)\right)$. Recall that it is an $\left(L(Y) \times k^{*}\right)$-orbit and by construction, for each point $\left(i, p, \eta, Y^{\prime}\right)$ of the orbit, we have a diagram

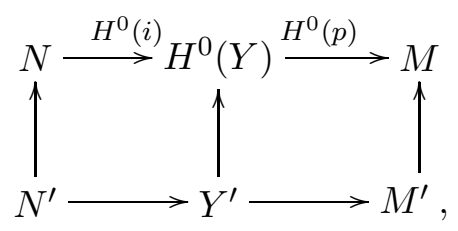


where $N^{\prime}$ is the preimage of $Y^{\prime}$ under $H^{0}(i)$ and $M^{\prime}$ the image of $Y^{\prime}$ under $H^{0}(p)$. We have a morphism of short exact sequences

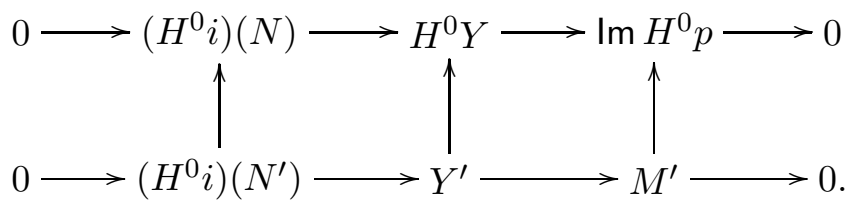

Thus, if we fix the triangle

$$
N \stackrel{i}{\longrightarrow} Y \stackrel{p}{\longrightarrow} M \stackrel{\eta}{\longrightarrow} S N
$$

then the possible submodules $Y^{\prime}$ form an affine space endowed with a simply transitive action by

$$
\operatorname{Hom}_{k Q}\left(H^{0}(p) Y^{\prime},\left(H^{0} i\right)(N) /\left(H^{0} i\right)\left(N^{\prime}\right)\right)=\operatorname{Hom}_{k Q}\left(M^{\prime}, N / N^{\prime}\right),
$$

where we have the last equality because $H^{0}(p)\left(Y^{\prime}\right)=M^{\prime}$ and $\operatorname{ker}\left(H^{0} i\right) \subset N^{\prime} \subset N$. Thus the space does not depend on the choice of a point $\left(i, p, \eta, Y^{\prime}\right)$ in the orbit. Moreover, the set of the possible straight lines

$$
[\eta] \in \mathbb{P}\left(\operatorname{Ext}_{\mathcal{C}_{Q}}^{1}(M, N) \cap \operatorname{Im} \beta\right) \subset \mathbb{P} \operatorname{Ext}^{1}(M, N)
$$

is the complement of the hyperplane defined by $\phi(? \cdot \eta)=0$ inside

$$
\mathbb{P}\left(\operatorname{Ext}_{\mathcal{C}_{Q}}^{1}(M, N) \cap \operatorname{Im} \beta\right) .
$$

Thus these $\eta$ also form an affine space. Therefore, the variety $p_{1}^{-1}\left([\varepsilon], M^{\prime}, N^{\prime}\right)$ is an extension of affine spaces by Lemma 3 .

We prove part b). Let $\left(i, p, \eta, Y^{\prime}\right)$ be a point of an orbit in $R_{2}$ and $\left([\eta], M^{\prime}, N^{\prime}\right)$ in $p_{1}\left(p_{2}^{-1}\left([\eta], Y^{\prime}\right)\right)$. Then, $N^{\prime}:=H^{0}(i)^{-1}\left(H^{0}\left(Y^{\prime}\right)\right)$ and $M^{\prime}:=H^{0}(p)\left(H^{0}\left(Y^{\prime}\right)\right)$ only depend on the choice of the orbit. Thus the set $p_{1} p_{2}^{-1}\left([\eta], Y^{\prime}\right)$ is parametrized by the $[\varepsilon]$ with $\phi(\eta \varepsilon) \neq 0$. These form an affine space inside $\mathbb{P} \operatorname{Ext}_{\mathcal{C}_{Q}}^{1}(M, N)$

We prove part c). Consider the diagram of $k Q$-modules

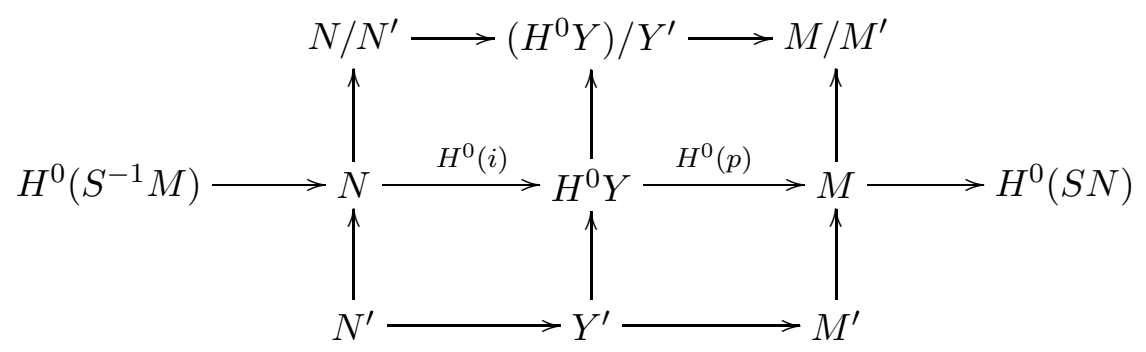

Since $N^{\prime}$ is the preimage of $Y^{\prime}$ under $H^{0}(i)$, the kernels of $N^{\prime} \rightarrow Y^{\prime}$ and $H^{0}(i)$ are isomorphic. We denote both by $K$. Dually, since $M^{\prime}$ is the image of $Y^{\prime}$ under $H^{0}(p)$, the cokernels of $\left(H^{0} Y\right) / Y^{\prime} \rightarrow M / M^{\prime}$ and of $H^{0}(p)$ are isomorphic. We denote both by $C$. Then in the Grothendieck group of $k Q$-modules, we have the following equalities

$$
\tau\left(N^{\prime}\right)+\tau\left(M^{\prime}\right)=\tau\left(Y^{\prime}\right)+\tau(K) \text { and } N / N^{\prime}+M / M^{\prime}=\left(H^{0} Y\right) / Y^{\prime}+C .
$$

Therefore we have

$$
\tau N^{\prime}-N+N^{\prime}+\tau M^{\prime}-M+M^{\prime}=\tau\left(Y^{\prime}\right)+\tau(K)-\left(H^{0} Y\right) / Y^{\prime}-C .
$$

In the notation of the proposition, it remains to be shown that

$$
x \underline{\operatorname{dim}} \tau(K)-\underline{\operatorname{dim}} C=X_{S P_{Y}} .
$$


Now in fact, it is easy to see that $X_{S P_{i}}=x^{I_{i}}$, where $I_{i}$ is the injective module associated to $i$. Hence, for each projective $k Q$-module $P$,

$$
X_{S P}=x^{\nu P},
$$

where $\nu$ is the Nakayama functor. So, what we have to prove is the equality

$$
\tau(K)-C=\nu P_{Y}
$$

in the Grothendieck group of $k Q$-modules. For this, we first note that by the triangle

$$
N \rightarrow Y \rightarrow M \rightarrow S N
$$

of $\mathcal{C}_{Q}$, the module $K$ is a quotient of $H^{0}\left(S^{-1} M\right)=H^{0}\left(\tau^{-1} M\right)$. Since $M$ is indecomposable non injective, $\tau^{-1} M$ is still a module so that $K$ is a quotient of $\tau^{-1} M$ and $\tau K$ a quotient of $M$. In particular, $\tau K$ is still a module and $\tau K=H^{0}(\tau K)$. Thus it suffices to prove that

$$
H^{0}(\tau K)-C=\nu P_{Y}
$$

in the Grothendieck group of $k Q$-modules. For this, we first note that we have a triangle

$$
N \stackrel{H^{0}(i)}{\longrightarrow} H^{0} Y \longrightarrow \operatorname{cok}\left(H^{0} i\right) \oplus S K \longrightarrow S N
$$

in $\mathcal{D}^{b}(k Q)$ and thus in $\mathcal{C}_{Q}$. Secondly, we have a split triangle

$$
S P_{Y} \longrightarrow Y \longrightarrow H^{0} Y \stackrel{0}{\longrightarrow} S^{2} P_{Y}
$$

in $\mathcal{C}_{Q}$; and thirdly, we have the triangle

$$
N \rightarrow Y \rightarrow M \rightarrow S N
$$

in $\mathcal{C}_{Q}$. Note that $H^{0} i$ is the composition of the morphism $N \rightarrow Y$ with the projection $Y \rightarrow H^{0} Y$. If we form the octahedron associated with this composition, the three triangles we have just mentioned appear among its faces, as well as a new triangle, namely

$$
S P_{Y} \longrightarrow M \longrightarrow \operatorname{cok}\left(H^{0} i\right) \oplus S K \longrightarrow S^{2} P_{Y} .
$$

Note that $S^{2} P_{Y}=\nu P_{Y}$ by the Calabi-Yau property. If we apply $H^{*}$ to this triangle, we obtain the exact sequence of $k Q$-modules

$$
0 \longrightarrow M \longrightarrow \operatorname{cok}\left(H^{0} i\right) \oplus H^{0}(\tau K) \longrightarrow \nu P_{Y} \longrightarrow H^{0}(\tau M)
$$

Since $M$ is an indecomposable module, $\tau M$ is either an indecomposable non injective module or zero. The image of $\nu P_{Y} \rightarrow \tau M=H^{0} \tau M$ is injective (as a quotient of an injective module). Hence it is zero and we get an exact sequence

$$
0 \longrightarrow M \longrightarrow \operatorname{cok}\left(H^{0} i\right) \oplus H^{0}(\tau K) \longrightarrow \nu P_{Y} \longrightarrow 0
$$

In the Grothendieck group, this yields

$$
0=M-\operatorname{cok}\left(H^{0} i\right)-H^{0}(\tau K)+\nu P_{Y}=C-H^{0}(\tau K)+\nu P_{Y}
$$

and this is what we had to prove. 
3.6. We give here some examples which illustrate Theorem 2

\section{Example 1.}

Suppose that $M$ and $N$ are indecomposable objects such that $\operatorname{dim} \operatorname{Ext}_{\mathcal{C}}^{1}(N, M)=1$. As in [6] let $B$ and $B^{*}$ be the unique objects such that there exist non split triangles $M \rightarrow B \rightarrow$ $N \rightarrow S M$ and $N \rightarrow B^{*} \rightarrow M \rightarrow S N$. In this case, we have

$$
\operatorname{Ext}^{1}(N, M)_{B}=k^{*}, \operatorname{Ext}^{1}(M, N)_{B^{*}}=k^{*} .
$$

The cluster multiplication theorem then asserts that $X_{N} X_{M}=X_{B}+X_{B^{*}}$. Note that in this particular case, this formula was a conjecture of [6] and is since a theorem of [10], [5]. Example 2.

If $Q$ is the following quiver of type $\mathrm{A}_{2}$ :

$$
1 \longleftarrow 2 \text {. }
$$

Set $M=S_{1} \oplus S_{1}, N=S_{2} \oplus S_{2}$. If $Y$ is an object such that $\mathbb{P} \operatorname{Ext}^{1}(N, M)_{Y}$ is not empty then $Y$ is either $S_{1} \oplus P_{2} \oplus S_{2}$ or $P_{2} \oplus P_{2}$ and it is an easy exercise to prove that the cardinality of $\mathbb{P} \operatorname{Ext}^{1}(N, M)_{Y}$ on $\mathbb{F}_{q}$ is respectively $q^{2}+2 q+1$ and $q\left(q^{2}-1\right)$. In a dual way, if $Y$ is an object such that $\mathbb{P} \operatorname{Ext}^{1}(M, N)_{Y}$ is not empty then $Y$ is either $S_{1} \oplus S_{2}$ or 0 and the cardinality of $\mathbb{P E x t}^{1}(N, M)_{Y}$ on $\mathbb{F}_{q}$ is respectively $q^{2}+2 q+1$ and $q\left(q^{2}-1\right)$.

The cluster multiplication theorem gives:

$$
X_{N} X_{M}=X_{S_{1} \oplus P_{2} \oplus S_{2}}+X_{S_{1} \oplus S_{2}} .
$$

Then, applying again the formula - note that we now in the case of the previous exampleyields:

$$
X_{N} X_{M}=X_{2 P_{2}}+2 X_{P_{2}}+1 \text {. }
$$

Note that this can be easily verified by taking squares in $X_{S_{2}} X_{S_{1}}=X_{P_{2}}+1$.

\section{Example 3.}

We give an example where the two indecomposable objects $M$ and $N$ are such that their first extension space has dimension 2 .

We consider the following quiver $Q$ of type $\mathrm{D}_{4}$ :

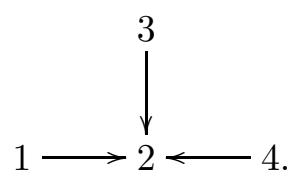

Set $\alpha_{i}=\underline{\operatorname{dim}}\left(S_{i}\right)$. Let $R, S, T, U$ be the indecomposable $k Q$-modules with respective dimension vectors $\alpha_{2}+\alpha_{3}+\alpha_{4}, \alpha_{1}+\alpha_{2}+\alpha_{4}, \alpha_{1}+\alpha_{2}+\alpha_{3}, \alpha_{1}+2 \alpha_{2}+\alpha_{3}+\alpha_{4}$. Consider the injective module $M=I_{2}$ and the simple module $N=S_{2}$. If $Y$ is an object such that $\mathbb{P} \operatorname{Ext}^{1}(M, N)_{Y}$ is not empty then $Y$ is either $U, R \oplus P_{1}, S \oplus P_{3}$ or $T \oplus P_{4}$ and the cardinality of $\mathbb{P x t}^{1}(M, N)_{Y}$ on $\mathbb{F}_{q}$ is respectively $q-2,1,1$ and 1 . Symmetrically, if $Y$ is an object such that $\mathbb{P} \operatorname{Ext}^{1}(N, M)_{Y}$ is not empty then $Y$ is either $S P_{2}, S P_{1}+S_{1}, S P_{3}+S_{3}$ or $S P_{4}+S_{4}$ and the cardinality of $\mathbb{P} \operatorname{Ext}^{1}(N, M)_{Y}$ on $\mathbb{F}_{q}$ is respectively $q-2,1,1$ and 1 . Hence, the cluster multiplication theorem gives

$2 X_{N} X_{M}=-X_{U}+X_{P_{1}} X_{R}+X_{P_{3}} X_{S}+X_{P_{4}} X_{T}-X_{S P_{2}}+X_{S P_{1}} X_{S_{1}}+X_{S P_{3}} X_{S_{3}}+X_{S P_{4}} X_{S_{4}}$.

Applying the formula again gives

$$
2 X_{N} X_{M}=-X_{U}+3\left(X_{U}+1\right)-X_{S P_{2}}+3\left(X_{S P_{2}}+1\right),
$$

and finally

$$
X_{N} X_{M}=X_{U}+3+X_{S P_{2}}
$$




\section{Finite Cluster algebras And Positivity}

4.1. In order to go further, we have to recall some terminology on cluster algebras. More precise and complete information can be found in 14 .

Let $n \leq m$ be two positive integers. We fix the coefficient field $\mathcal{F}_{0}=\mathbb{Q}\left(u_{n+1}, \ldots u_{m}\right)$ and the ambient field $\mathcal{F}=\mathbb{Q}\left(u_{1}, \ldots, u_{m}\right)$, where the $u_{i}$ 's are indeterminates. Let $\mathbf{x}$ be a free generating set of $\mathcal{F}$ over $\mathcal{F}_{0}$ and let $\tilde{B}=\left(b_{i j}\right)$ be an $m \times n$ integer matrix such that the submatrix $B=\left(b_{i j}\right)_{1 \leq i, j \leq n}$ is antisymmetric. Such a pair $(\mathbf{x}, \tilde{B})$ is called a seed.

Let $(\mathbf{x}, \tilde{B})$ be a seed and let $x_{j}, 1 \leq j \leq n$, be in $\mathbf{x}$. We define a new seed as follows. Let $x_{j}^{\prime}$ be the element of $\mathcal{F}$ defined by the exchange relation:

$$
x_{j} x_{j}^{\prime}=\prod_{b_{i j}>0} x^{b_{i j}}+\prod_{b_{i j}<0} x^{-b_{i j}}
$$

where, by convention, we have $x_{i}=u_{i}$ for $i>n$. Set $\mathbf{x}^{\prime}=\mathbf{x} \cup\left\{x_{j}^{\prime}\right\} \backslash\left\{x_{j}\right\}$. Let $\tilde{B}^{\prime}$ be the $m \times n$ matrix given by

$$
b_{i k}^{\prime}= \begin{cases}-b_{i k} & \text { if } i=j \text { or } k=j \\ b_{i k}+\frac{1}{2}\left(\left|b_{i j}\right| b_{j k}+b_{i j}\left|b_{j k}\right|\right) & \text { otherwise. }\end{cases}
$$

Then a result of Fomin and Zelevinsky asserts that $\left(\mathbf{x}^{\prime}, \tilde{B}^{\prime}\right)$ is a seed. It is called the mutation of the seed $(\mathbf{x}, \tilde{B})$ in the direction $x_{j}$. We consider all the seeds obtained by iterated mutations. The free generating sets occurring in the seeds are called clusters, and the variables they contain are called cluster variables. By definition, the cluster algebra $\mathcal{A}(\mathbf{x}, \tilde{B})$ associated to the seed $(\mathbf{x}, \tilde{B})$ is the $\mathbb{Z}\left[u_{n+1}, \ldots, u_{m}\right]$-subalgebra of $\mathcal{F}$ generated by the set of cluster variables. The Laurent phenomenon, see [12, asserts that the cluster variables are Laurent polynomials with integer coefficients in the $x_{i}, 1 \leq i \leq m$. So, we have $\mathcal{A}(\mathbf{x}, B) \subset \mathbb{Z}\left[x_{1}^{ \pm 1}, \ldots, x_{m}^{ \pm 1}\right]$.

Except in section 5.4 we will be concerned with cluster algebras such that $n=m$, i.e. $\tilde{B}=B$. Note that an antisymmetric matrix $B$ defines a quiver $Q=Q_{B}$ with vertices corresponding to its rows (or columns) and which has $b_{i j}$ arrows from the vertex $i$ to the vertex $j$ whenever $b_{i j}>0$. The cluster algebra associated to the seed $(\mathbf{x}, B)$ will be also denoted by $\mathcal{A}(Q)$.

An important result of [14] asserts that a cluster algebra is finite, i.e. has a finite number of cluster variables, if and only if there exists a seed associated to a quiver of simply laced Dynkin type. In this case, the Dynkin type is unique.

Now fix a quiver $Q$ of simply laced Dynkin type. Then, by [8], the $\mathbb{Z}$-module generated by the variables $X_{M}$, where $M$ runs over the set of objects of $\mathcal{C}_{Q}$, is an algebra; it is the cluster algebra $\mathcal{A}(Q)$ and the cluster variables are the $X_{M}$ 's, where $M$ runs through the indecomposable objects of $\mathcal{C}_{Q}$.

An object $M$ of $\mathcal{C}$ is called exceptional if it has no selfextensions, i.e. $\operatorname{Ext}^{1}(M, M)=0$. An object $T$ of $\mathcal{C}$ is a tilting object if it is exceptional, multiplicity free, and has the following maximality property: if $M$ is an indecomposable object such that $\operatorname{Ext}^{1}(M, T)=0$, then $M$ is a direct factor of a direct sum of copies of $T$. Note that a tilting object can be identified with a maximal set of indecomposable objects $T_{1}, \ldots, T_{n}$ such that $\operatorname{Ext}^{1}\left(T_{i}, T_{j}\right)=0$ for all $i, j$.

In view of $\left[8\right.$, the main result of [6] can be stated as follows: the map $X_{\text {? }}: M \mapsto X_{M}$ induces a bijection from the set of tilting objects to the set of clusters of $\mathcal{A}(Q)$.

4.2. Here we prove a positivity theorem that was conjectured in [14], see also [8].

Theorem 3. For any object $M$ of $\mathcal{C}_{Q}$, the variable $X_{M}$ is in $\mathbb{Z}_{\geq 0}\left[u_{i}^{ \pm 1}\right]$. 
Proof. It is sufficient to prove that for any $M$ in $\bmod k Q$, and for any $e$ in $\mathbb{N}^{n}$, we have $\chi_{c}\left(\operatorname{Gr}_{e}(M)\right) \geq 0$. For this, we recall the construction of the (classical) Hall algebra $\mathcal{H}(Q)$ of $\bmod (Q)$ : The algebra $\mathcal{H}(Q)$ is the vector space with basis $\left\{e_{M}\right\}_{M}$, where $M$ runs through the set of isoclasses of finite-dimensional $k Q$-modules. The multiplication rule on $\mathcal{H}(Q)$ is given by

$$
e_{M} * e_{N}=\sum_{X} P_{M, N}(1) e_{X},
$$

where $P_{M, N}^{X}$ is the Hall polynomial defined by

$$
P_{M, N}^{X}(q)=\left.\#\{Y, Y \in \operatorname{Gr}(X), Y \simeq N, X / Y \simeq M\}\right|_{\mathbb{F}_{q}} .
$$

It is known from 27] that $\mathcal{H}(Q)$ is an associative algebra, isomorphic to the enveloping algebra $U(\mathfrak{n})$ of a maximal nilpotent subalgebra $\mathfrak{n}$ of the semisimple Lie algebra $\mathfrak{g}$ associated to the Dynkin diagram underlying $Q$. Via the isomorphism $\mathcal{H}(Q) \simeq U(\mathfrak{n})$, the basis $\left\{e_{M}\right\}_{M}$ is identified with a Poincaré-Birkoff-Witt basis of $U(\mathfrak{n})$ (in the sense of [21]).

For any dimension vector $e$, set

$$
b_{e}=\sum_{\underline{\operatorname{dim}}(N)=e} e_{N} \in \mathcal{H}(Q) .
$$

Then we have

$$
b_{e^{\prime}} b_{e}=\sum_{\underline{\operatorname{dim}}\left(N^{\prime}\right)=e^{\prime}} e_{N^{\prime}} \sum_{\underline{\operatorname{dim}}(N)=e} e_{N}=\sum_{\underline{\operatorname{dim}} M=e+e^{\prime}}\left(\sum_{\underline{\operatorname{dim}} N^{\prime}=e^{\prime}, \underline{\operatorname{dim}} N=e} P_{N^{\prime}, N}^{M}(1)\right) e_{M} .
$$

Hence,

$$
b_{e^{\prime}} b_{e}=\sum_{\underline{\operatorname{dim}} m=e+e^{\prime}} \chi_{c}\left(\mathrm{Gr}_{e}(M)\right) e_{M},
$$

by Lemma 4. Now by [21, 7.3], for any dimension vector $e$, the element $b_{e}$ is in Lusztig's canonical basis of $U(\mathfrak{n})$, when the quantification parameter $q$ is equal to 1 . Moreover, by [22, par. 14], the product of two elements of the canonical basis has positive coefficients in its expansion in the canonical basis. Finally, by [21, 7.11], an element of the canonical basis has positive coefficients in its expansion in the PBW-base $\left\{e_{M}\right\}$. Hence we have $\chi_{c}\left(\mathrm{Gr}_{e}(M)\right) \geq 0$.

We can have more by noting that the element $b_{e}$ of the proof is the element of the canonical basis associated to the dense orbit of the moduli space of dimension vector $e$. This easily implies that $\chi_{c}\left(\operatorname{Gr}_{e}(M)\right)>0$ if $\operatorname{Gr}_{e}(M) \neq \emptyset$. It would be interesting to prove that the variety $\operatorname{Gr}_{e}(M)$ has a cellular decomposition and to find a combinatorial way to calculate its Euler characteristic.

As a particular case of the theorem, we obtain the

Corollary 1. For any quiver $Q$ of simply laced Dynkin type, the cluster variables of $\mathcal{A}(Q)$ are Laurent polynomials in the variables $x_{i}$ with positive integer coefficients.

We can also generalize Fomin and Zelevinky's denominator theorem [14, see also 9 , Theorem 3.6], to any quiver $Q$ of simply laced Dynkin type:

Corollary 2. Let $M$ be an indecomposable $k Q$-module and set $\underline{\operatorname{dim}} M=\sum_{i} m_{i} \underline{\operatorname{dim}} S_{i}$. Then the denominator of $X_{M}$ as an irreducible fraction of integral polynomials in the variables $u_{i}$ is $\prod u_{i}^{m_{i}}$.

Proof. By 3.1 and the positivity theorem, $X_{M}$ is a linear combination with positive integer coefficients of terms $\prod u_{i}^{n_{i}}, n_{i} \in \mathbb{Z}$. These terms are indexed by the set of dimension vectors 
of submodules $N$ of $M$, and for each submodule $N$, we have, by the Serre duality formula, that

$$
n_{i}=-\left\langle N, S_{i}\right\rangle-\left\langle S_{i}, M / N\right\rangle .
$$

So, it is sufficient to prove that

1. for all $i$, we have $\left\langle N, S_{i}\right\rangle+\left\langle S_{i}, M / N\right\rangle \leq(\underline{\operatorname{dim}} M)_{i}$ and

2 . for all $i$, there exists a submodule $N$ such that the equality holds.

First recall that for each module $X$, we have $\left\langle X, I_{i}\right\rangle=\left\langle P_{i}, X\right\rangle=(\underline{\operatorname{dim}} X)_{i}$. Now, as $\bmod k Q$ is hereditary, the injective resolution of $S_{i}$ yields

$$
\left\langle N, S_{i}\right\rangle \leq\left\langle N, I_{i}\right\rangle \text {. }
$$

Dually, we have

$$
\left\langle S_{i}, M / N\right\rangle \leq\left\langle P_{i}, M / N\right\rangle .
$$

Adding both inequalities and using the formula above gives the first point. Now fix a vertex $i$ of the quiver and let $J$ be the set of vertices $j$ such that there exists a path from $i$ to $j$. Define the subspace $N$ of $M$ to be the sum of the subspaces $e_{j} M, j \in J$, where $e_{j}$ is the idempotent associated with $j$. Then, by construction, $N$ is a submodule of $M$ with the following properties: a) $(\underline{\operatorname{dim}} N)_{j}=0$ if there is a path $j \rightarrow i$, b) $(\underline{\operatorname{dim}} M / N)_{j}=0$ if there is a path $i \rightarrow j$. Considering the injective resolution

$$
0 \rightarrow S_{i} \rightarrow I_{i} \rightarrow I \rightarrow 0
$$

we obtain the equality $\left\langle N, S_{i}\right\rangle=\left\langle N, I_{i}\right\rangle-\langle N, I\rangle=\left\langle N, I_{i}\right\rangle$, by a). Dually, property b) implies that $\left\langle S_{i}, M / M_{i}\right\rangle=\left\langle P_{i}, M / M_{i}\right\rangle$. So we obtain the equality $\left\langle M_{i}, S_{i}\right\rangle+\left\langle S_{i}, M / M_{i}\right\rangle=$ $(\underline{\operatorname{dim}} M)_{i}$ as required.

\section{Filtrations And Bases}

As in the previous section, we assume that $Q$ is a quiver of simply laced Dynkin type. Recall that the elements of the generating set $X_{M}, M \in \operatorname{obj}\left(\mathcal{C}_{Q}\right)$, of $\mathcal{A}(Q)$ can be written

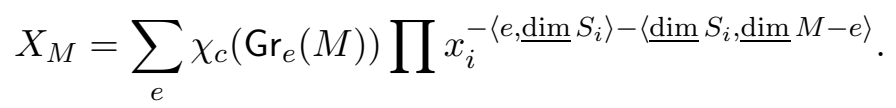

We will show that this formula provides 'good' filtrations for finite cluster algebras.

5.1. Fix a quiver $Q$ of simply laced Dynkin type and let $B$ be the antisymmetric matrix such that $Q=Q_{B}$. We can view $B$ as an endomorphism of $\mathrm{G}_{0}(\bmod k Q)$ endowed with the basis $\underline{\operatorname{dim}} S_{i}, 1 \leq i \leq n$.

Lemma 6. We have $B e=\sum_{i}\left(\left\langle e, \underline{\operatorname{dim}} S_{i}\right\rangle-\left\langle\underline{\operatorname{dim}} S_{i}, e\right\rangle\right) \underline{\operatorname{dim}} S_{i}$.

Proof. Recall that $B=\left(b_{i j}\right)$ with

$$
b_{i j}= \begin{cases}1 & \text { if } i \rightarrow j \\ -1 & \text { if } j \rightarrow i \\ 0 & \text { otherwise }\end{cases}
$$

Hence,

$$
B\left(\underline{\operatorname{dim}} S_{j}\right)=\sum_{i \rightarrow j} \underline{\operatorname{dim}} S_{i}-\sum_{j \rightarrow i} \underline{\operatorname{dim}} S_{i}
$$


Note now that

$$
\left\langle\underline{\operatorname{dim}} S_{j}, \underline{\operatorname{dim}} S_{i}\right\rangle-\left\langle\underline{\operatorname{dim}} S_{i}, \underline{\operatorname{dim}} S_{j}\right\rangle= \begin{cases}1 & \text { if } i \rightarrow j \\ -1 & \text { if } j \rightarrow i \\ 0 & \text { otherwise. }\end{cases}
$$

This proves the lemma.

We need the

Lemma 7. Let $M$ be an indecomposable $k Q$-module and let $N$ be a proper submodule of M. Then, $B(\underline{\operatorname{dim}} N) \neq 0$.

Proof. Suppose that $N \subset M$ and $\left\langle\underline{\operatorname{dim}} N, \underline{\operatorname{dim}} S_{i}\right\rangle-\left\langle\underline{\operatorname{dim}} S_{i}, \underline{\operatorname{dim}} N\right\rangle=0$ for all $i$. This implies that for any $k Q$-module $X$, we have $\langle N, X\rangle-\langle X, N\rangle=0$.

Suppose first that $N$ has no injective component. Then, $N$ has an injective hull $I$, with the following property: $[N, I]^{1}=0=[I, N]^{0}$. Hence,

$$
\langle N, I\rangle-\langle N, I\rangle \geq[N, I]^{0}>0,
$$

which contradicts the above formula.

Suppose that $N$ has a non zero injective component $J$. Then since $N$ is a proper submodule of $M$, the module $J$ is a proper direct factor of $M$, in contradiction with the assumption that $M$ is indecomposable.

For any Laurent polynomial $X$ in the set of variables $\left\{x_{i}\right\}$, the support $\operatorname{supp}(X)$ of $X$ is by definition the set of points $\lambda=\left(\lambda_{1}, \ldots, \lambda_{n}\right)$ of $\mathbb{Z}^{n}$ such that the $\lambda$-component, i.e. the coefficient in $\prod_{i} x^{\lambda_{i}}$, of $X$ is non zero. For any point $\lambda$ in $\mathbb{Z}^{n}$, identified with $\mathrm{G}_{0}(\bmod k Q)$, let $C_{\lambda}$ be the convex cone with vertex $\lambda$, and whose edge vectors are generated by $B\left(\underline{\operatorname{dim}} S_{i}\right)$. The previous lemma easily implies the following proposition.

Proposition 5. Fix an indecomposable object $M$ of $\mathcal{C}_{Q}$, and let $M=M_{0} \oplus S P_{M}$ be its decomposition as in 2.4. Then, $\operatorname{supp}\left(X_{M}\right)$ is in $C_{\lambda_{M}}$ with $\lambda_{M}:=\left(-\left\langle\underline{\operatorname{dim}} S_{i}, \underline{\operatorname{dim}} M_{0}\right\rangle+\right.$ $\left.\left\langle\underline{\operatorname{dim}} P_{M}, \underline{\operatorname{dim}} S_{i}\right\rangle\right)$. Moreover, the $\lambda_{M}$-component of $X_{M}$ is 1 .

5.2. The following proposition rephrases a result of [23].

Proposition 6. The map $\lambda_{\text {? }}: \mathcal{C} \rightarrow \mathbb{Z}^{n}, M \mapsto \lambda_{M}$ is surjective. Any fiber of a point in $\mathbb{Z}^{n}$ contains a unique exceptional object. The cones generated by the images of tilting objects provide a complete simplicial fan.

Proof. We first describe the exceptional objects of $\mathcal{C}$. For any $M$ in $\operatorname{obj}(\mathcal{C})$, we denote by $I_{M}$ the set of $i$ such that $P_{i}$ is a component of $P_{M}$. The following fact is clear :

The object $M=M_{0} \oplus S P_{M}$ is exceptional if and only if $M_{0}$ is exceptional and $\left(\underline{\operatorname{dim}} M_{0}\right)_{i}=$ 0 for any $i$ in $I_{M}$.

Recall now that for any dimension vector $d$ in $\mathrm{G}_{0}(\bmod k Q)$, there exists a unique exceptional module $M_{d}$ such that $\underline{\operatorname{dim}}\left(M_{d}\right)=d$.

Let $E$ be the set of exceptional modules. It decomposes into the disjoint union $E=\coprod E_{I}$, where $I$ runs over the set of partitions of $\{1, \ldots, n\}$ and where $E_{I}:=\left\{M \in E, I_{M}=I\right\}$.

For any object $M=M_{0} \oplus\left(\oplus_{i} m_{i} S P_{i}\right)$, we set $\underline{\operatorname{dim}}(M)=\underline{\operatorname{dim}}\left(M_{0}\right)-\left(m_{1}, \ldots, m_{n}\right)$.

On the one hand, it is known by [23] that the cones generated by the images under dim of tilting objects of $\mathcal{C}$ provide a complete simplicial fan in $\mathbb{Z}^{n}$. On the other hand, by the assertion above, $\underline{\operatorname{dim}}$ provides a bijection from $E$ to $\mathbb{Z}^{n}$, and via this bijection, the map $\lambda_{\text {? }}$ is piecewise linear - the domains of linearity are the $E_{I}$ 's. Moreover, on $E_{I}$, the matrix of 
$\lambda$ is triangular and the diagonal components are

$$
d_{i}=\left\{\begin{array}{ll}
1 & \text { if } i \in I \\
-1 & \text { if } i \notin I
\end{array} .\right.
$$

This proves the proposition.

5.3. Under the following hypothesis on $Q$, we will now define a filtration of the cluster algebra $\mathcal{A}(Q) \subset \mathbb{Z}\left[x_{1}^{ \pm 1}, \ldots, x_{n}^{ \pm 1}\right]$.

HYPOTHESIS: There exists a form $\epsilon$ on $\mathbb{Z}^{n}$ such that

$$
\epsilon\left(B_{Q} \underline{\operatorname{dim}} S_{i}\right) \in \mathbb{Z}_{<0}, \text { for all } i \text {. }
$$

Note that for any Dynkin diagram except $\mathrm{A}_{1}$, there exists an orientation $Q$ satifying our hypothesis. Indeed, let $Q_{a l t}$ be an alternating quiver. Then the matrix $B_{a l t}=\left(b_{i j}\right)$ associated to this quiver satisfies $b_{i j} \geq 0$ if $i$ is a source, and $b_{i j} \leq 0$ if $i$ is a sink. Moreover, each row of $B_{\text {alt }}$ is non zero. So we can take any form $\epsilon$ whose coordinates in the dual basis of $\mathbb{Z}^{n}$ satisfy $\epsilon_{i}<0$ if $i$ is a source and $\epsilon_{i}>0$ if $i$ is a sink. Note also that we have

$$
B e=\sum_{i}\left\langle e+\tau e, \underline{\operatorname{dim}} S_{i}\right\rangle \underline{\operatorname{dim}} S_{i}
$$

for all $e \in \mathbb{Z}^{n}$ so that the above hypothesis holds iff the image of the positive cone of $\mathrm{G}_{0}(\bmod k Q)$ under the map $\tau+\mathbf{1}$ is strictly contained in a halfspace.

For any $n$ in $\mathbb{Z}$, set

$$
F_{n}=\left(\oplus_{\epsilon(\mu) \leq n} \mathbb{Z} \prod x_{i}^{\mu_{i}}\right) \cap \mathcal{A}(Q) .
$$

Using Proposition 5 and Proposition [6] we obtain:

Proposition 7. The set $\left(F_{n}\right)_{n \in \mathbb{Z}}$ defines a filtration of $\mathcal{A}(Q)$. The graded algebra associated to this filtration is isomorphic to $\mathbb{Z}\left[u_{1}^{ \pm 1}, \ldots, u_{n}^{ \pm 1}\right]$.

Proof. As we have $F_{n} F_{m} \subset F_{n+m}$, the sequence $\left(F_{n}\right)$ is a filtration of $\mathcal{A}(Q)$. Moreover, Proposition 5 implies that for any indecomposable module $M$, we have

$$
\operatorname{gr} X_{M}=\operatorname{gr} \prod_{i} u_{i}^{\left(\lambda_{M}\right)_{i}}
$$

The result then follows from Proposition 6

This implies

Corollary 3. For any Dynkin quiver, the set $\mathcal{B}:=\left\{X_{M}, M \in \operatorname{obj}(\mathcal{C})\right.$, $\left.\operatorname{Ext}^{1}(M, M)=0\right\}$ of variables corresponding to exceptional objets of the category $\mathcal{C}$ is a $\mathbb{Z}$-basis of the cluster algebra $\mathcal{A}(Q)$.

Proof. This is obviously true for a quiver of type $\mathrm{A}_{1}$. Now, for any quiver, we have $\mathcal{A}(Q)=\mathcal{A}\left(Q^{\prime}\right)$ for some quiver $Q^{\prime}$ which satisfies the hypothesis above. In this case, the proposition together with formula 5.1 imply that $\mathcal{B}$ is $\mathbb{Z}$-free.

Let us prove now that $\mathcal{B}$ generates $\mathcal{A}(Q)$ as a $\mathbb{Z}$-module. We first define a degeneration ordering $\prec_{e}$ in obj $(\mathcal{C})$. Let $M, M^{\prime}$ be objects of $\mathcal{C}$. We say that there is an elementary degeneration $M^{\prime} \prec_{e} M$ if $M-M^{\prime}$ is an elementary vector in $K_{0}^{\text {split }}(\mathcal{C})$. We have

Lemma 8. Let $M, M^{\prime}$ be objects of $\mathcal{C}$.

a) We have $M^{\prime} \prec_{e} M$ iff there are decompositions $M=L \oplus U \oplus V$ and $M^{\prime}=L \oplus E$ where $U$ and $V$ are indecomposable and $E$ is the middle term of a non split triangle

$$
U \longrightarrow E \longrightarrow V \longrightarrow S U
$$


b) If we have $M^{\prime} \prec_{e} M$, then

$$
0 \leq \operatorname{dim} \operatorname{Ext}^{1}\left(M^{\prime}, M^{\prime}\right)<\operatorname{dim} \operatorname{Ext}^{1}(M, M) .
$$

Proof. a) If the condition holds, then $M^{\prime}-M$ equals the elementary vector $U+V-E$. Conversely, if $M^{\prime}-M$ is an elementary vector, we have $M^{\prime}-M=U+V-E$ where $U$, $V$ are indecomposable and $E$ is the middle term of a non split triangle as in the assertion. Then we have $M^{\prime}+E=M+U+V$. It follows from Proposition 1 and Lemma 1 that $U$ and $V$ are not direct factors of $E$. Moreover, they are non isomorphic since no indecomposable has selfextensions. Thus, the object $M^{\prime}$ decomposes as the sum of some $L$ and $U \oplus V$ so that we obtain $L+U+V+E=M+U+V$ and $L+E=M$.

b) Let $U, V$ and $L$ be as in a). For any objects $N, N^{\prime}$ of $\mathcal{C}$, set $\left[N, N^{\prime}\right]^{1}=\operatorname{dim}_{\operatorname{Ext}} t_{\mathcal{C}}^{1}\left(N, N^{\prime}\right)$. We view [?, ?] ${ }^{1}$ as a symmetric bilinear form on $K_{0}^{\text {split }}(\mathcal{C})$. For any object $N$ of $\mathcal{C}$, the long exact sequence obtained by applying $\operatorname{Hom}(?, S N)$ to the triangle

$$
U \longrightarrow E \longrightarrow V \stackrel{e}{\longrightarrow} S U
$$

shows that we have

$$
[E, N]^{1} \leq[U, N]^{1}+[V, N]^{1} .
$$

Moreover, for $N=U$, we have the strict inequality

$$
[E, U]<[U, U]^{1}+[V, U]^{1}
$$

since in the sequence

$$
\operatorname{Hom}(V, S U) \rightarrow \operatorname{Hom}(E, S U) \rightarrow \operatorname{Hom}(U, S U),
$$

the first map is not injective: its kernel contains $e$. By the inequality $(*)$, we have

$$
[L, L]^{1}+2[L, E]^{1} \leq[L, L]^{1}+2[L, U+V]
$$

and it only remains to be shown that

$$
[E, E]^{1}<[U+V, U+V]^{1} .
$$

For this, we note that by the above inequalities, we have

$$
[E, E]^{1} \leq[E, U]^{1}+[E, V]^{1},[E, V]^{1} \leq[U, V]^{1}+[V, V]^{1} \text { and }[E, U]^{1}<[U, U]^{1}+[V, U]^{1} \text {. }
$$

Let us finish the proof of the corollary. It remains to be proved that each $X_{M}$ is in $\mathbb{Z} \mathcal{B}$. If $M$ is indecomposable, then $M$ is exceptional and hence is in $\mathcal{B}$. If $M$ is not indecomposable, say $M=M^{\prime} \oplus M^{\prime \prime}$, then by the cluster multiplication theorem and the lemma above, $X_{M}$ expands into a $\mathbb{Q}$-linear combination of terms $X_{Y}$ for objects $Y$ such that $0 \leq \operatorname{Ext}^{1}(Y, Y)<\operatorname{Ext}^{1}(M, M)$. By induction, we obtain that $X_{M}$ is in $\mathbb{Q} \mathcal{B}$. As the coefficients $\chi_{c}\left(\operatorname{Gr}_{e}(M)\right)$ in the cluster variable formula are integers, we obtain, using induction on the filtration, that $X_{M}$ is in $\mathbb{Z} \mathcal{B}$.

5.4. In this section, we consider the more general case of finite cluster algebras associated to a rectangular $m \times n$ matrix $\tilde{B}$. We want to prove that our construction provides a toric degeneration of the spectrum of finite cluster algebras.

Let $B$ be the antisymmetric submatrix associated to $\tilde{B}$. We have a projection $\pi: \mathcal{A}(\tilde{B}) \rightarrow$ $\mathcal{A}(B)$ such that $u_{i} \mapsto u_{i}$, if $1 \leq i \leq n$, and $u_{i} \mapsto 1$, if $n+1 \leq i \leq m$. This projection gives a one-to-one correspondence between the cluster variables of the two cluster algebras. For any indecomposable object $M$ of $\mathcal{C}$, we denote by $\tilde{X}_{M}$ the cluster variable such that $\pi\left(\tilde{X}_{M}\right)=X_{M}$. We fix a quiver $Q$ as in the previous section and we suppose without loss 
of generality that $B=B_{Q}$. Let $F_{n}$ be the filtration of $\mathcal{A}(B)$ constructed from $Q$. We now consider the filtration $\tilde{F}_{n}=\pi^{-1}\left(F_{n}\right), n \in \mathbb{Z}$, induced by $\pi$ from the filtration $F_{n}$.

Theorem 4. The graded algebra $\operatorname{gr} \mathcal{A}(\tilde{B})$ associated to the filtration $\tilde{F}_{n}$ is isomorphic to a subalgebra of $\mathbb{Z}\left[u_{i}^{ \pm 1}, 1 \leq i \leq m\right]$ generated by a finite set of unitary monomials.

Proof. For any $z$ in $\mathcal{A}(\tilde{B})$, we denote by $\operatorname{gr} z$ the corresponding element in the graded algebra $\operatorname{gr} \mathcal{A}(\tilde{B})$. It is sufficient to prove that for any indecomposable object $M$ of $\mathcal{C}, \operatorname{gr} \tilde{X}_{M}$ is a unitary monomial in the gr $u_{i}$ 's. This is true for $M=S P_{i}$ as in this case, gr $\tilde{X}_{M}$ is the monomial $u_{i}$. Now, we make an induction with the help of the Hom-ordering $\preceq$ in ind $\bmod k Q$. By the exchange relation as in [8, 3.4], we have

$$
\tilde{X}_{\tau(M)} \tilde{X}_{M}=p \prod_{i} \tilde{X}_{B_{i}}+q
$$

where $p, q$ are unitary monomials in the $u_{i}^{\prime} s, n+1 \leq i \leq m$. In this relation, $\tau(M)$ and $B_{i}$ are indecomposable objects which verify the induction hypothesis. Suppose that $\operatorname{gr} \prod \tilde{X}_{B_{i}}$ and 1 have the same degree in $\operatorname{gr} \mathcal{A}(\tilde{B})$. Then, the monomial $\operatorname{gr} \prod X_{B_{i}}$ and 1 have the same degree in $\operatorname{gr} \mathcal{A}(B)$. But this would imply that the coefficient of the monomial $\operatorname{gr} X_{M}$ is not 1, in contradiction with Proposition 5 . Hence, $\operatorname{gr} \prod \tilde{X}_{B_{i}}$ and 1 do not have the same degree in $\operatorname{gr} \mathcal{A}(\tilde{B})$. This implies that either $\operatorname{gr} \tilde{X}_{\tau(M)} \operatorname{gr} \tilde{X}_{M}=\operatorname{gr} p \operatorname{gr} \prod \tilde{X}_{B_{i}}$ or $\operatorname{gr} \tilde{X}_{\tau(M)} \operatorname{gr} \tilde{X}_{M}=\operatorname{gr} q$. In both cases, the induction process is proved.

This is a classical corollary of the theorem, see [7].

Corollary 4. The spectrum of a finite cluster algebra has a toric degeneration.

5.5. With the help of the basis of Corollary 3, we can reformulate Theorem 2, Actually, we can give a complete realization of the cluster algebra $\mathcal{A}(Q)$ from the cluster category $\mathcal{C}_{Q}$

We recall the degeneration ordering $\prec_{e}$ in $\operatorname{obj}(\mathcal{C})$ defined for the proof of Lemma 8 Suppose that there is an elementary degeneration $M^{\prime} \preceq_{e} M$ with elementary vector $Z_{i}+$ $Z_{j}-Y_{i j}$. Then, by the lemma 8 we can define the ratio

$$
r\left(M, M^{\prime}\right):=c z_{i} z_{j} / \operatorname{dim} \operatorname{Ext}^{1}(M, M),
$$

where $c$ is the multiplicity number associated to the elementary vector, and where $z_{i}$, resp. $z_{j}$, are the multiplicity of $Z_{i}$, resp. $Z_{j}$, in $M$. Let $\prec$ be the ordering generated by $\prec_{e}$.

Note the surprising fact that in the category $\mathcal{C}$, the composition of elementary degenerations can again be an elementary degeneration.

Note that, by [5.2, any chain of elementary degenerations descending from an object $M$ is finite.

For any pair of objects $M, N$ of $\mathcal{C}$ and for any chain from $N$ to $M$

$$
\Gamma: N=M_{0} \prec_{e} M_{1} \prec_{e} \ldots \prec_{e} M_{k}=M,
$$

we set

$$
r(M, N, \Gamma)=\prod_{i=1}^{k} r\left(M_{i}, M_{i-1}\right), \quad r(M, N)=\sum_{\Gamma} r(M, N, \Gamma),
$$

where $\Gamma$ runs through the chains from $N$ to $M$.

We define the exceptional Hall algebra of $\mathcal{C}$ to be the rational vector space $\mathcal{H}_{\text {exc }}(\mathcal{C})$ of $\mathbb{Q}$-valued functions on the isomorphism classes of exceptional objects of $\mathcal{C}$ endowed with 
the multiplication given by

$$
\chi_{M} * \chi_{N}=\sum_{K} r(M \oplus N, K) \chi_{K},
$$

where the sum runs over the isomorphism classes of exceptional objects $K$ and $\chi_{K}$ denotes the characteristic function. From Theorem 2 and section 3.2. we easily deduce the

Theorem 5. The exceptional Hall algebra $\mathcal{H}_{\text {exc }}\left(\mathcal{C}_{Q}\right)$ is an associative $\mathbb{Q}$-algebra with unit element $\chi_{0}$. The map $\chi_{M} \mapsto X_{M}$ induces an isomorphism between the exceptional Hall algebra $\mathcal{H}_{\text {exc }}\left(\mathcal{C}_{Q}\right)$ and the cluster algebra $\mathcal{A}_{Q}$.

\section{Conjectures}

6.1. The results in [8] and in this article are concerned with finite cluster algebras, with a fixed seed corresponding to a Dynkin quiver. We conjecture some generalizations for any seed.

Fix a quiver $Q$ of Dynkin type and a tilting object $T$ of $\mathcal{C}_{Q}$. Consider the so-called tilted algebra $A_{T}:=\operatorname{End}_{\mathcal{C}}(T)^{o p p}$ and the category $\bmod A_{T}$ of finite dimensional $A_{T}$-modules.

We consider the form

$$
\langle N, M\rangle=\operatorname{dim} \operatorname{Hom}(N, M)-\operatorname{dim} \operatorname{Ext}^{1}(N, M), \quad N, M \in \bmod A_{T} .
$$

Remark that in general this form does not descend to the Grothendieck group of the category mod $A_{T}$. One defines the antisymmetrized form:

$$
\langle N, M\rangle_{a}=\langle N, M\rangle-\langle M, N\rangle, \quad N, M \in \bmod A_{T} .
$$

We know that there exists a seed $\left(\mathbf{x}_{T}, B_{T}\right)$ of the cluster algebra $\mathcal{A}(Q)$ associated to the tilting object $T, c f$. 6]. Moreover, by [4], the set $\operatorname{ind}\left(\bmod A_{T}\right)$ is in bijection with the set of cluster variables which do not belong to $\mathbf{x}_{T}$.

Conjecture 1. The form $\langle,\rangle_{a}$ descends to the Grothendieck group $\mathrm{G}_{0}\left(\bmod A_{T}\right)$. Its matrix for the basis $\left.\underline{\underline{\operatorname{dim}}} S_{i}\right)$ is $B_{T}$.

Set $\mathbf{x}_{T}=\left\{x_{1}, \ldots, x_{n}\right\}$. The following conjecture describes the bijection explained above. It can be seen as a generalization of Theorem 3.4 of [8] .

Conjecture 2. To any indecomposable module $M$ in $\bmod A_{T}$, we assign

$$
X_{M}:=\sum_{e} \chi_{c}\left(\operatorname{Gr}_{e}(M)\right) \prod_{i} x_{i}^{\left(B_{T} e\right)_{i}-\left\langle S_{i}, M\right\rangle} .
$$

Then the set $\left\{X_{M}, M \in \operatorname{ind} \bmod A_{T}\right\}$ is exactly the set of cluster variables which do not belong to $\mathbf{x}_{T}$.

Via a conjectural extension of theorem 3 this conjecture would yield positivity properties.

6.2. As before, fix a tilting object $T=\oplus_{i=1}^{n} T_{i}$ of $\mathcal{C}_{Q}$, with $T_{i}$ indecomposable. By the discussion above, the set ind $(\mathcal{C})$ can be seen as a disjoint union

$$
\operatorname{ind}(\mathcal{C})=\operatorname{ind} \bmod A_{T} \coprod\left\{T_{i}, 1 \leq i \leq n\right\} .
$$

Hence, as in 2.4 each object $M$ of $\mathcal{C}$ has a unique decomposition $M=M_{0} \oplus T_{M}$, where $M_{0}$ is in $\bmod A_{T}$ and where $T_{M}$ is a direct factor of a sum of copies of $T$.

Suppose that Conjecture 2 is true. Then, for any object $M=M_{0} \oplus\left(\oplus_{i} m_{i} T_{i}\right)$, we can define the variable 


$$
X_{M}:=\sum_{e} \chi_{c}\left(\operatorname{Gr}_{e}\left(M_{0}\right)\right) \prod_{i} x_{i}^{\left(B_{T} e\right)_{i}-\left\langle S_{i}, M\right\rangle+m_{i}} .
$$

Define the map $\lambda_{?}: \mathcal{C} \rightarrow \mathbb{Z}^{n}$ to be given by $M \mapsto\left(\left\langle S_{i}, M\right\rangle-m_{i}\right)$.

Conjecture 3. The cones generated by the images of tilting objects under $\lambda_{\text {? }}$ provide a complete simplicial fan.

Note that if we replace the map $\lambda_{\text {? }}$ by the dimension vector map, we obtain a complete fan which is in general not simplicial.

6.3. We finish with a positivity conjecture which can be seen as an analogue of Lusztig's positivity theorem, cf. [22, for the dual canonical basis.

Conjecture 4. For any object $M$ and any exceptional object $K$ of $\mathcal{C}$, the integer $r(M, K)$ is non negative.

In particular, the conjecture implies that the coefficients in Theorem 5 are positive. Note that the rational numbers $r(M, N, \Gamma)$ defined in section 5 can be negative.

\section{ApPENDIX ON CONSTRUCTIBILITY}

We present a general proof for the constructibility of the sets $\operatorname{Ext}^{1}(M, N)_{Y}$ in a triangulated category with finitely many isoclasses of indecomposables.

Let $k$ be a field and $\mathcal{T}$ a $k$-linear triangulated category with suspension functor $S$ such that

- all Hom-spaces in $\mathcal{T}$ are finite-dimensional,

- each indecomposable of $\mathcal{T}$ has endomorphism ring $k$,

- each object is a finite direct sum of indecomposables,

- $\mathcal{T}$ has Serre duality, i.e. there is an equivalence $\nu: \mathcal{T} \rightarrow \mathcal{T}$ such that we have

$$
D \operatorname{Hom}(X, ?) \stackrel{\sim}{\rightarrow} \operatorname{Hom}(?, \nu X)
$$

for each $X \in \mathcal{T}$, where $D$ denotes the functor $\operatorname{Hom}_{k}(?, k)$.

It is not hard to show that the last condition is a consequence of the first three. The conditions imply that $\mathcal{T}$ has Auslander-Reiten triangles and that the Auslander-Reiten translation $\tau$ is given by $S^{-1} \nu$.

For objects $X, Y, Z$ of $\mathcal{T}$, let $\operatorname{Hom}(X, Y)_{Z}$ be the set of morphisms $f: X \rightarrow Y$ such that there is a triangle

$$
X \stackrel{f}{\longrightarrow} Y \longrightarrow Z \longrightarrow S X
$$

Proposition 8. The set $\operatorname{Hom}(X, Y)_{Z}$ is constructible.

Proof. Recall that a split triangle is a triangle which is a direct sum of triangles one of whose three morphisms is an isomorphism. Let us call a triangle minimal if it does not have a non zero split triangle as a direct factor. A triangle

$$
X \stackrel{f}{\longrightarrow} Y \longrightarrow Z \longrightarrow S X \text {. }
$$

is minimal iff, in the category of morphisms, $f$ does not admit non zero factors of the following forms

$$
U \stackrel{\mathbf{1}_{U}}{\longrightarrow} U, U \longrightarrow 0,0 \longrightarrow U
$$

Let us call such morphisms $f$ minimal. We now proceed by induction on the sum $s$ of the numbers of indecomposable modules occurring in the decompositions of $X$ and $Y$ into 
indecomposables. Clearly the assertion holds if $s=0$ i.e. $X=Y=0$. Let us suppose $s>0$. Then $\operatorname{Hom}(X, Y)_{Z}$ is the disjoint union of the set $M$ of morphisms $f$ such that the triangle

$$
X \stackrel{f}{\longrightarrow} Y \longrightarrow Z \longrightarrow S X
$$

is minimal and of the set $M^{\prime}$ of morphisms admitting a non zero direct factor of one of the above forms. The set $M^{\prime}$ is the union of orbits under $\operatorname{Aut}(X) \times \operatorname{Aut}(Y)$ of morphisms of the forms

$$
f^{\prime} \oplus \mathbf{1}_{U}: X^{\prime} \oplus U \rightarrow Y^{\prime} \oplus U,\left[f^{\prime}, 0\right]: X^{\prime} \oplus U \rightarrow Y^{\prime},\left[f^{\prime}, 0\right]^{t}: X^{\prime} \rightarrow Y^{\prime} \oplus U,
$$

where $U$ is non zero and $f^{\prime}$ runs through the sets $\operatorname{Hom}\left(X^{\prime}, Y^{\prime}\right)_{Z^{\prime}}$ for suitable $X^{\prime}, Y^{\prime}, Z^{\prime}$, of which there are only a finite number. It therefore follows from the induction hypothesis that $M^{\prime}$ is constructible. It remains to be shown that the set $M$ is constructible. We work in the category $\bmod \mathcal{T}$ of finitely presented functors on $\mathcal{T}$ with values in the category of $k$-vector spaces. It is an abelian category. Its projective objects are the representable functors $\widehat{U}=\operatorname{Hom}(?, U)$ and these are also the injective objects. If $U$ is indecomposable in $\mathcal{T}$ and $S_{U}$ is the simple top of the indecomposable projective $\widehat{U}$, then $S_{U}$ admits the minimal projective presentation

$$
\widehat{E_{U}} \stackrel{\widehat{p_{U}}}{\longrightarrow} \widehat{U} \longrightarrow S_{U} \longrightarrow 0
$$

where

$$
\tau U \longrightarrow E_{U} \stackrel{p_{U}}{\longrightarrow} U \longrightarrow S \tau U
$$

is an Auslander-Reiten triangle. Moreover, $S_{U}$ is also the simple socle of the indecomposable injective

$$
\widehat{S \tau U}=\widehat{\nu U} .
$$

If $f: X \rightarrow Y$ is a minimal morphism, then the morphisms

$$
\widehat{X} \rightarrow \operatorname{im}(\widehat{f}), \operatorname{im}(\widehat{f}) \rightarrow \widehat{Y}
$$

induced by $f$ are a projective cover and an injective hull, respectively. Moreover, if $f$ is minimal and

$$
X \stackrel{f}{\longrightarrow} Y \stackrel{g}{\longrightarrow} Z \longrightarrow S X
$$

is a triangle, then $g$ is also minimal, so that $\widehat{Z}$ is an injective hull of $\operatorname{im}(\widehat{g})=\operatorname{cok}(\widehat{f})$. Therefore, the multiplicity $m_{U}$ of an indecomposable object $\nu U$ in the decomposition of $Z$ into indecomposables equals the multiplicity of the simple $S_{U}$ in the socle of $\operatorname{cok}(\widehat{f})$. Since $U$ has endomorphism algebra $k$, this also holds for $S_{U}$ and the multiplicity $m_{U}$ equals

$$
\operatorname{dim} \operatorname{Hom}\left(S_{U}, \operatorname{cok}(\widehat{f})\right) \text {. }
$$

Now we have projective presentations

$$
\widehat{E_{U}} \stackrel{\widehat{p_{U}}}{\longrightarrow} \widehat{U} \longrightarrow S_{U} \longrightarrow 0
$$

and

$$
\widehat{X} \stackrel{\widehat{f}}{\longrightarrow} \widehat{Y} \longrightarrow \operatorname{cok}(\widehat{f}) \longrightarrow 0
$$


Thus, we can compute the space $\operatorname{Hom}\left(S_{U}, \operatorname{cok}(\widehat{f})\right)$ as the quotient of the space of morphisms

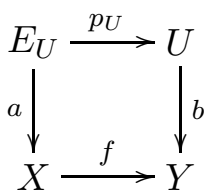

modulo the subspace formed by the morphisms of the form $(a, b)=\left(c p_{U}, f c\right)$ for some morphism $c: U \rightarrow X$. The condition

$$
\operatorname{dim} \operatorname{Hom}\left(S_{U}, \operatorname{cok}(\widehat{f})\right)=m_{U}
$$

then translates into conditions on the ranks of the linear maps

$$
(a, b) \mapsto b p_{U}-f a \text { and } c \mapsto\left(c p_{U}, f c\right) .
$$

Clearly, the $f \in \operatorname{Hom}(X, Y)$ satisfying these rank conditions for all indecomposables $U$ form a constructible subset. The intersection of this subset with the complement of $M^{\prime}$ is still constructible (since $M^{\prime}$ is) and clearly equals $M$. So $M$ is constructible.

\section{REFERENCES}

[1] A. Berenstein, S. Fomin, A. Zelevinsky. Cluster algebras. III. Upper bounds and double Bruhat cells. Duke Math. J. 126 (2005), no. 1, 1-52.

[2] A. Białynicki-Birula. Some theorems on actions of algebraic groups. Ann. of Math. 98 (1973), 480-497.

[3] S. Brenner, M. C. R. Butler, The equivalence of certain functors occurring in the representation theory of Artin algebras and species. Journal of the LMS 14 (1976), no. 1, 183-187.

[4] A.B. Buan, R.J. Marsh, I. Reiten. Cluster tilted algebras. math.RT/0402075

[5] A.B. Buan, R.J. Marsh, I. Reiten. Cluster mutation via quiver representations. math.RT/0412077

[6] A.B. Buan, R.J. Marsh, M. Reineke, I. Reiten, G. Todorov. Tilting theory and cluster combinatorics. math.RT/0402054

[7] P. Caldero. Toric degenerations of Schubert varieties. Transform. Groups 7 (2002), no. 1, 51-60.

[8] P. Caldero, F. Chapoton. Cluster algebras as Hall algebras of quiver representations. math.RT/0410184

[9] P. Caldero, F. Chapoton, R. Schiffler. Quivers with relations arising from clusters ( $\mathrm{A}_{n}$ case). math.RT/0410184 accepted in Trans. Amer. Math. Soc.

[10] P. Caldero, F. Chapoton, R. Schiffler. Quivers with relations and cluster tilted algebras. math.RT/0411238

[11] P. Caldero, R. Schiffler. Rational smoothness of varieties of representations for quivers of Dynkin type. Ann. Inst. Fourier (Grenoble) 54 (2004), no. 2, 295-315.

[12] S. Fomin, A. Zelevinsky. Cluster algebras. I. Foundations. J. Amer. Math. Soc. 15 (2002), no. 2, 497-529.

[13] V.V. Fock, A.B. Goncharov. Cluster ensembles, quantization and the dilogarithm. math.AG/0311245

[14] S. Fomin, A. Zelevinsky. Cluster algebras. II. Finite type classification. Invent. Math. 154 (2003), no. $1,63-121$.

[15] P. Gabriel. Auslander-Reiten sequences and representation-finite algebras. Representation theory, I (Proc. Workshop, Carleton Univ., Ottawa, Ont., 1979). LNM 831, Springer, 1980.

[16] C. Geiss, B. Leclerc, J. Schröer. Rigid modules over preprojective algebras. math.RT/0503324

[17] M. Gekhtman, M. Shapiro, A. Vainshtein. Cluster algebras and Poisson geometry. Mosc. Math. J. 3 (2003), no. 3, 899-934, 1199.

[18] J. A. Green. Hall algebras, hereditary algebras and quantum groups. Inv. Math. 120 (1995), no. 2, 361-377.

[19] D. Happel, Triangulated categories in the representation theory of finite-dimensional algebras. London Mathematical Society Lecture Note Series, 119. Cambridge University Press, Cambridge, 1988.

[20] B. Keller. Triangulated orbit categories. math.RT/0503240

[21] G. Lusztig. Canonical bases arising from quantized enveloping algebras J. of AMS, Vol. 3, Number 2, (1990), 447-498.

[22] G. Lusztig. Introduction to quantum groups. Progress in Mathematics 110, Birkhäuser, Boston, 1993.

[23] R. Marsh, M. Reineke, A. Zelevinsky. Generalized associahedra via quiver representations. Trans. Amer. Math. Soc. 355 (2003), no. 10, 4171-4186. 
[24] L. Peng, J. Xiao. Triangulated categories and Kac-Moody algebras. Invent. Math. 140 (2000), no.3, 563-603.

[25] Reineke, M. Counting rational points of quiver moduli. preprint.

[26] C. Riedtmann. Lie algebras generated by indecomposables. Journal of algebra 170, 526-546, 1994.

[27] C. M. Ringel. Hall polynomials for the representation-finite hereditary algebras. Advances in Mathematics, 84 (1990), 137-178.

[28] C. M. Ringel. Hall algebras. Banach Center Publications, 26 (1990), 433-447.

[29] J. Scott. Ph.D. thesis. Northeastern University (2003).

[30] B. Toën. Derived Hall Algebras. math.QA/0501343

[31] B. Toën, M. Vaquié. Moduli of objects in dg-categories. math.AG/0503269

[32] J.-L. Verdier, Catégories dérivées, état 0, in SGA 4.5, 1977, Lecture notes 569, Springer-Verlag, 1977, 262-308.

Institut Camille Jordan, Université Claude Bernard Lyon I, 69622 Villeurbanne Cedex, FRANCE

E-mail address: caldero@igd.univ-lyon1.fr

UFR de mathématiques, Université Denis Diderot - Paris 7, 2 Place Jussieu, 75251 Paris Cedex 05, France

E-mail address: keller@math.jussieu.fr 